\title{
A stabilized finite element method for generalized stationary incompressible flows
}

\author{
Ramon Codina \\ Universitat Politècnica de Catalunya, Jordi Girona 1-3, Edifici C1, 08034 Barcelona, Spain
}

Received 15 December 1998; received in revised form 7 April 2000

\begin{abstract}
In this paper, we describe a finite element formulation for the numerical solution of the stationary incompressible Navier-Stokes equations including Coriolis forces and the permeability of the medium. The stabilized method is based on the algebraic version of the sub-grid scale approach. We first describe this technique for general systems of convection-diffusion-reaction equations and then we apply it to the linearized flow equations. The important point is the design of the matrix of stabilization parameters that the method has. This design is based on the identification of the stability problems of the Galerkin method and a scaling of variables argument to determine which coefficients must be included in the stabilization matrix. This, together with the convergence analysis of the linearized problem, leads to a simple expression for the stabilization parameters in the general situation considered in the paper. The numerical analysis of the linearized problem also shows that the method has optimal convergence properties. (C) 2001 Elsevier Science B.V. All rights reserved.
\end{abstract}

\section{Introduction}

When the Navier-Stokes equations for an incompressible viscous fluid are written in a rotating frame of reference two new terms appear, namely, the centrifugal force and the Coriolis force. The former can be written as $\frac{1}{4} \omega \times(\boldsymbol{\omega} \times \boldsymbol{r})$, where $\boldsymbol{\omega}$ is twice the velocity of rotation of the frame of reference and $\boldsymbol{r}$ the vector of position of the particles referred to this system. This term can be included in the vector of body forces and offers no additional difficulty. On the other hand, the Coriolis force is $\boldsymbol{\omega} \times \boldsymbol{u}$, where $\boldsymbol{u}$ is the velocity field referred to the rotating reference. This term has to be added to the Navier-Stokes operator. Another term that has to be included in this operator is the one coming from the medium resistance to the flow when the permeability of this medium is finite. In the simplest model considered here, this term has the form $\sigma \boldsymbol{u}$, where $\sigma \geqslant 0$ is the inverse of the permeability coefficient (in the case of anisotropic media $\sigma$ is a secondorder tensor).

Another term that we shall include in the flow equations is a penalty parameter in the continuity equation, which then will be written as $\varepsilon p+\nabla \cdot \boldsymbol{u}=0, p$ being the kinematic fluid pressure and $\varepsilon \geqslant 0$ given. This parameter $\varepsilon$ will be assumed small in the formulation presented herein. Precise upper bounds for it will be specified in terms of the stabilization parameters of the formulation.

E-mail address: ramon.codina@upc.es; URL: http://www.rmee.upc.es/homes/codina (R. Codina). 
Taking into account all the effects described, the problem to be solved in the steady-state case is

$$
\begin{aligned}
& (\boldsymbol{u} \cdot \nabla) \boldsymbol{u}+\frac{1}{2}(\nabla \cdot \boldsymbol{u}) \boldsymbol{u}+\boldsymbol{\omega} \times \boldsymbol{u}-v \Delta \boldsymbol{u}+\sigma \boldsymbol{u}+\nabla p=\boldsymbol{f} \text { in } \Omega, \\
& \varepsilon p+\nabla \cdot \boldsymbol{u}=0 \quad \text { in } \Omega, \\
& \boldsymbol{u}=\mathbf{0} \quad \text { on } \Gamma,
\end{aligned}
$$

where $f$ is the vector of body forces, including also the centrifugal force, $v$ the kinematic viscosity of the fluid and $\Omega$ is the computational domain, with $\Gamma=\partial \Omega$ its boundary. For simplicity, we consider only homogeneous Dirichlet boundary conditions. Observe also that we have written the convective term in its skew-symmetric form. For truly incompressible flows, the term $\frac{1}{2}(\nabla \cdot \boldsymbol{u}) \boldsymbol{u}$ can be omitted, but when $\varepsilon>0$, it helps to ensure that the problem is well posed.

There are several numerical difficulties associated with problem (1)-(3) when the standard Galerkin finite element method is used. The first of them is classical and concerns the compatibility of the finite element spaces for the velocity and the pressure. It is well known that they have to satisfy the so-called inf-sup or Babuška-Brezzi stability condition (see, e.g., [1]). There is also the possibility of using the same interpolation for both the velocity and the pressure by modifying the discrete variational form.

The second numerical problem is also well known. It concerns the relative importance of the viscous and convective terms. When the latter becomes important the standard Galerkin formulation fails and numerical oscillations occur.

Another difficulty encountered when one tries to solve the problem with small values of the viscosity is the presence of spurious oscillations due to the Coriolis force. This problem was identified and discussed for the Stokes case (i.e., without the nonlinear convective term) in [2]. These oscillations are due to the pressure

Which may be understoo incompressibility were not borhood of the boundary pioportional to the unknow norm, though it is
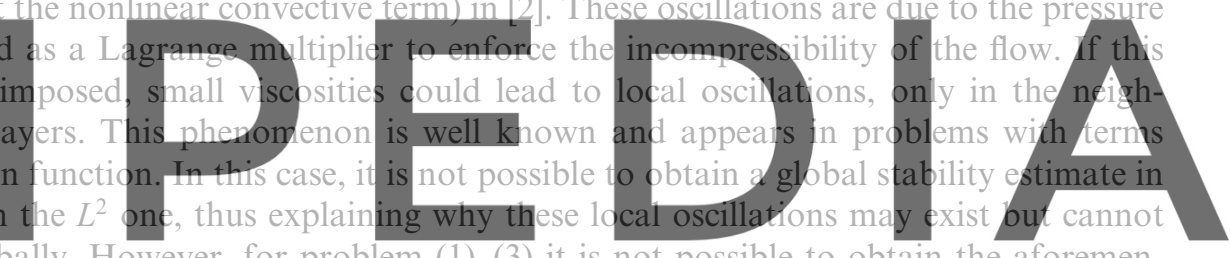

deteriorate the solution globally. However, for problem (1)-(3) it is not possible to obtain the aforemen-

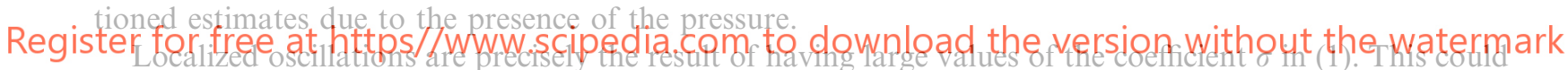

occur if the permeability of the medium where the flow takes place is very low. The situation is similar to what happens when there is an important reactive term in the linear scalar convection-diffusion-reaction equation. Stabilized methods for this kind of problem have been studied, e.g., in [3,4]. We shall see that the formulation presented here is in fact simpler than those proposed in these references (though we apply it to a much more complex case).

In this paper, we propose a stabilized finite element method for the problem that deals with all the numerical difficulties discussed, i.e., it allows to use equal velocity-pressure interpolation, it solves the instabilities due to dominant convective and Coriolis forces, and it is also able to deal with the case of large coefficients $\sigma$ in (1). Clearly, the idea of using a stabilized method able to deal with the pressure instability and convection dominated flows is old and is in fact the origin of the Galerkin/least-squares method (GLS) [5]. Extensions of this method specially oriented to the incompressible Navier-Stokes equations are presented, e.g., in [6-8], among others, and convergence analyses can be found already in $[9,10]$ and, more recently, in [11], where the full nonlinear problem is analyzed.

The method proposed here can be formulated starting from the sub-grid scale concept, an idea presented in [12] and described in Section 2 for systems of convection-diffusion-reaction equations. We use here a particular version of this stabilization technique. In Section 3, we apply it to the finite element approximation of Eqs. (1)-(3). The resulting numerical method depends on two algorithmic parameters, $\tau_{1}$ and $\tau_{2}$, that are designed from the analysis of what happens in a simple 1D problem [13]. However, this only serves as a motivation for the expression of these parameters. Once they are determined, a stability and convergence analysis can be conducted showing that the resulting method is stable and optimally convergent. This is done in Section 4 for the linearized problem. This analysis is not only a theoretical exercise, but 
clearly shows how $\tau_{1}$ and $\tau_{2}$ must behave. The expression we propose for them allows this analysis to be very simple, at least compared to the analyses that can be found, e.g., in [6] (for the linearized problem) or in [11], where neither the Coriolis forces nor the permeability of the medium are taken into account. In Section 5, we present some numerical results showing the good numerical performance of the stabilized formulation and finally we draw some conclusions in Section 6.

\section{Sub-grid scale approach}

When the Navier-Stokes equations are linearized, they lead to a system of convection-diffusion-reaction equations of the form

$$
\mathscr{L}(\boldsymbol{U}):=\frac{\partial}{\partial x_{i}}\left(\boldsymbol{A}_{i} \boldsymbol{U}\right)-\frac{\partial}{\partial x_{i}}\left(\boldsymbol{K}_{i j} \frac{\partial \boldsymbol{U}}{\partial x_{j}}\right)+\boldsymbol{S} \boldsymbol{U}=\boldsymbol{F} \quad \text { in } \Omega,
$$

where $\boldsymbol{U}$ and $\boldsymbol{F}$ are vectors of $n_{\text {unk }}$ unknowns and $\boldsymbol{A}_{i}, \boldsymbol{K}_{i j}$ and $\boldsymbol{S}$ are $n_{\mathrm{unk}} \times n_{\mathrm{unk}}$ matrices $\left(i, j=1, \ldots, n_{\mathrm{sd}}\right)$. The usual summation convention is implied in (4), with indices running from 1 to the number of space dimensions $n_{\mathrm{sd}}$. We shall refer to the terms of the left-hand side of this equation as the convective, the diffusive and the reactive term. The algebraic bilinear form associated to $\boldsymbol{K}_{i j}, i, j=1, \ldots, n_{\mathrm{sd}}$, is positivesemidefinite for the linearized Navier-Stokes equations, as we shall see. However, for the purpose of this section we can take it positive-definite. This allows us to consider the simplest Dirichlet boundary condition

$$
\boldsymbol{U}=\mathbf{0} \text { on } \Gamma \text {. }
$$

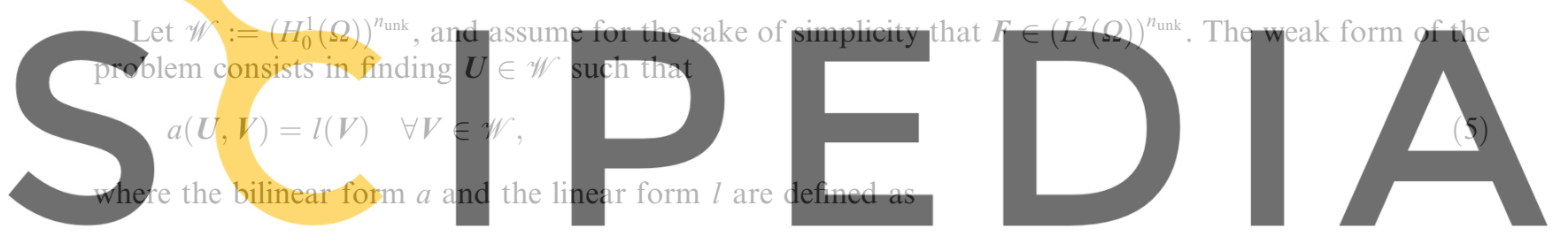

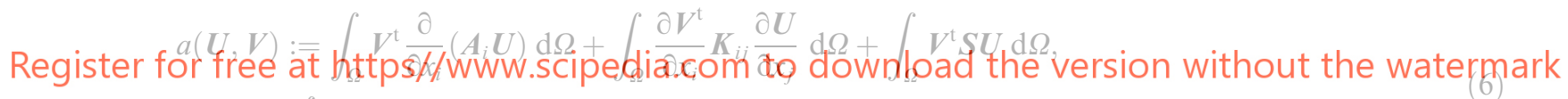

$$
l(V):=\int_{\Omega} V^{\mathrm{t}} F \mathrm{~d} \Omega
$$

The Galerkin finite element approximation of this problem is standard. If $\mathscr{W}_{h}$ is a finite element space to approximate $\mathscr{W}$, the discrete problem consists in finding $\boldsymbol{U}_{h} \in \mathscr{W}_{h}$ such that

$$
a\left(\boldsymbol{U}_{h}, \boldsymbol{V}_{h}\right)=l\left(\boldsymbol{V}_{h}\right) \quad \forall \boldsymbol{V}_{h} \in \mathscr{W}_{h} .
$$

It is well known that this formulation lacks stability when the diffusive terms are small, compared either to the convective or to the reactive terms. Several numerical methods have been proposed in order to overcome this misbehavior, such as the SUPG or the GLS methods [14,15]. In this section, we present a stabilized finite element method for this problem based on the sub-grid scale concept introduced in [12] (see also [16]).

Let us split the continuous space $\mathscr{W}$ as $\mathscr{W}=\mathscr{W}_{h} \oplus \tilde{\mathscr{W}}$, where $\tilde{\mathscr{W}}$ can be in principle any space to complete $\mathscr{W}_{h}$ in $\mathscr{W}$. We call it the space of sub-scales or sub-grid scales. The weak form of the continuous equation (5) can now be written as the system:

$$
\begin{aligned}
& a\left(\boldsymbol{U}_{h}, \boldsymbol{V}_{h}\right)+a\left(\tilde{\boldsymbol{U}}, \boldsymbol{V}_{h}\right)=l\left(\boldsymbol{V}_{h}\right) \quad \forall \boldsymbol{V}_{h} \in \mathscr{W}_{h}, \\
& a\left(\boldsymbol{U}_{h}, \tilde{\boldsymbol{V}}\right)+a(\tilde{\boldsymbol{U}}, \tilde{\boldsymbol{V}})=l(\tilde{\boldsymbol{V}}) \quad \forall \tilde{\boldsymbol{V}} \in \tilde{\mathscr{W}},
\end{aligned}
$$

where $\boldsymbol{U}=\boldsymbol{U}_{h}+\tilde{\boldsymbol{U}}$, with $\boldsymbol{U}_{h} \in \mathscr{W}_{h}, \tilde{\boldsymbol{U}} \in \tilde{\mathscr{W}}$. 
Let $n_{\mathrm{el}}$ be the number of elements of the finite element partition of the domain $\Omega$ and let $\Omega^{e}$ be the region ocuppied by the eth element, with boundary $\Gamma^{e}$. It is useful for the following to introduce the notation

$$
\int_{\Omega^{\prime}}:=\sum_{e=1}^{n_{\mathrm{el}}} \int_{\Omega^{e}}, \quad \int_{\partial \Omega^{\prime}}:=\sum_{e=1}^{n_{\mathrm{el}}} \int_{\Gamma^{e}} .
$$

We denote by $h^{e}$ the diameter of $\Omega^{e}$. The superscript $e$ in $h^{e}$ will be omitted if there is no possibility of confusion.

Let us assume that the solution of the continuous problem $\boldsymbol{U}$ is smooth. Integrating by parts within each element domain it is found that problem (7), (8) can be written as

$$
\begin{aligned}
& a\left(\boldsymbol{U}_{h}, \boldsymbol{V}_{h}\right)+\int_{\partial \Omega^{\prime}} \tilde{\boldsymbol{U}}^{\mathrm{t}} n_{i} \boldsymbol{K}_{i j} \frac{\partial \boldsymbol{V}_{h}}{\partial x_{j}} \mathrm{~d} \Gamma+\int_{\Omega^{\prime}} \tilde{\boldsymbol{U}}^{\mathrm{t}} \mathscr{L}^{*}\left(\boldsymbol{V}_{h}\right) \mathrm{d} \Omega=l\left(\boldsymbol{V}_{h}\right), \\
& \int_{\partial \Omega^{\prime}} \tilde{\boldsymbol{V}}^{\mathrm{t}} n_{i} \boldsymbol{K}_{i j} \frac{\partial}{\partial x_{j}}\left(\boldsymbol{U}_{h}+\tilde{\boldsymbol{U}}\right) \mathrm{d} \Gamma+\int_{\Omega^{\prime}} \tilde{\boldsymbol{V}}^{\mathrm{t}} \mathscr{L}(\tilde{\boldsymbol{U}}) \mathrm{d} \Omega=\int_{\Omega^{\prime}} \tilde{\boldsymbol{V}}^{\mathrm{t}}\left[\boldsymbol{F}-\mathscr{L}\left(\boldsymbol{U}_{h}\right)\right] \mathrm{d} \Omega
\end{aligned}
$$

for all $\boldsymbol{V}_{h} \in \mathbb{W}_{h}$ and $\tilde{\boldsymbol{V}} \in \tilde{\mathbb{W}}$, where $n_{i}$ is the ith component of the exterior normal to $\partial \Omega$ and $\mathscr{L}^{*}$ is the adjoint operator of $\mathscr{L}$ with homogeneous Dirichlet conditions, given by

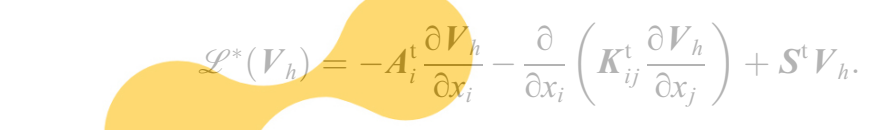

Since the normal component of the diffusive flux

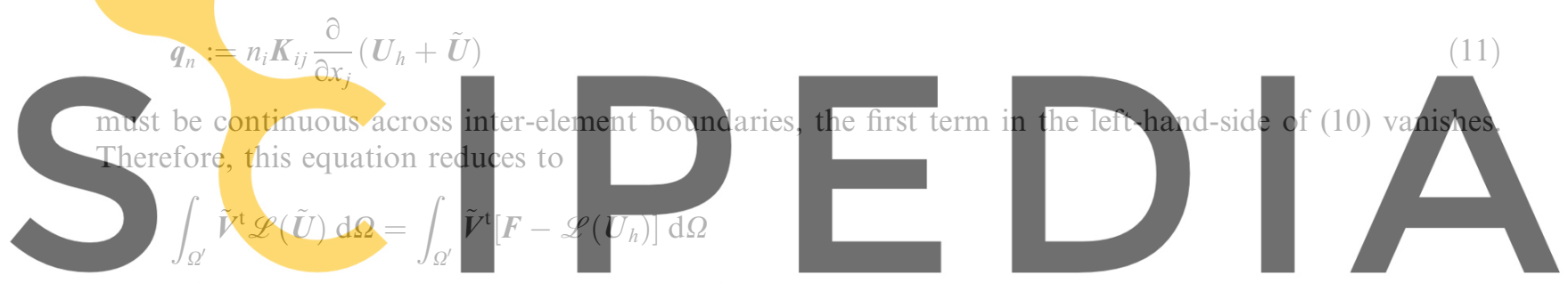

for all $\tilde{V} \in \tilde{\mathbb{W}}$, which is equivalent to finding $\tilde{U} \in \tilde{\mathbb{W}}$ such that

Register fQ2 free at https//, Www.scipedia.com to download the version without the watermark

$$
\tilde{\boldsymbol{U}}=\tilde{\boldsymbol{U}}_{\text {ske }} \quad \text { on } \partial \Omega^{e}
$$

for $e=1, \ldots, n_{\mathrm{el}}$, where $\boldsymbol{V}_{h, \text { ort }}$ is obtained from the condition that $\tilde{\boldsymbol{U}}$ must belong to $\tilde{\mathscr{W}}$ (and not to the whole space $\mathscr{W}$ ) and $\tilde{\boldsymbol{U}}_{\text {ske }}$ is a function defined on the element boundaries and such that the normal component of the diffusive flux is continuous across inter-element boundaries.

Problem (7), (8) is exactly equivalent to (9), (12), (13). The approximate problem is defined by the way in which problem (12), (13) is solved as well as by the way in which the functions $\boldsymbol{V}_{h \text { ort }}$ and $\tilde{\boldsymbol{U}}_{\text {ske }}$ are taken.

A particular case of the method described above is an algebraic approximation to the sub-scales [12]. The approximation of problem (12), (13) in this case is

$$
\tilde{\boldsymbol{U}} \approx \tau\left[\boldsymbol{F}-\mathscr{L}\left(\boldsymbol{U}_{h}\right)\right]
$$

where $\tau$ is an $n_{\text {unk }} \times n_{\text {unk }}$ matrix defined within each element domain that has to be determined. We shall refer to it as the matrix of stabilization parameters.

The approximation given by (14) has an implicit assumption on the function $\tilde{\boldsymbol{U}}_{\text {ske }}$ and the space $\tilde{\mathscr{W}}$, and therefore on the function $\boldsymbol{V}_{h \text {,ort }}$. In general, $\tilde{\boldsymbol{U}}$ will be discontinuous across inter-element boundaries, so that the fluxes given by (11) will not even be well defined. However, from (9) it is observed that, except for the boundary integral, only the component of $\tilde{\boldsymbol{U}}$ in $\mathscr{L}\left(\mathscr{W}_{h}\right)$ is needed, where $\mathscr{L}\left(\mathscr{W}_{h}\right)$ is the space of functions of the form $\mathscr{L}\left(\boldsymbol{V}_{h}\right)$, with $\boldsymbol{V}_{h} \in \mathscr{W}_{h}$. We may think of (14) as the approximation to this component.

To close the approximation, we neglect the inter-element boundary terms in (9), so that the problem that has to be solved is finally 


$$
a\left(\boldsymbol{U}_{h}, \boldsymbol{V}_{h}\right)+\int_{\Omega^{\prime}} \tilde{\boldsymbol{U}}^{\mathrm{t}} \mathscr{L}^{*}\left(\boldsymbol{V}_{h}\right) \mathrm{d} \Omega=l\left(\boldsymbol{V}_{h}\right)
$$

for all $\boldsymbol{V}_{h} \in \mathscr{W}_{h}$, with $\tilde{\boldsymbol{U}}$ given by (14). With all these assumptions we have arrived to the method proposed in [12] (for the scalar convection-diffusion equation) using different arguments. In particular, (14) was derived from an approximation to Green's function of the problem. This method was also considered in [17] and derived for the scalar diffusion-reaction equation in [18] by using bubble functions.

Even though the justification for the approximation (14) to $\tilde{U}$ is weak, the important point is the structure of the terms added to those coming from the Galerkin method in (15). It is this structure which will allow us to design a stabilized finite element method whose approximation properties are valid for all the values of the parameters of (1) (except for $\varepsilon$, which must be small, as it has been already said).

At this point it is important to remark that (15) may be obtained also if the bilinear form associated to $\boldsymbol{K}_{i j}, i, j=1, \ldots, n_{\mathrm{sd}}$, is only positive-semidefinite. The only thing that would change is the boundary conditions to be applied to $\tilde{\boldsymbol{U}}$ on the boundaries of the elements.

\section{Stabilized method for generalized incompressible flows}

\subsection{The linearized Navier-Stokes equations as a convection-diffusion-reaction system}

The previous stabilization method can be applied to problem (1)-(3) after identification of this as a system of convection-diffusion-reaction equations. The main point will be the design of the matrix of stabilization parameters appearing in $(14), \tau$.

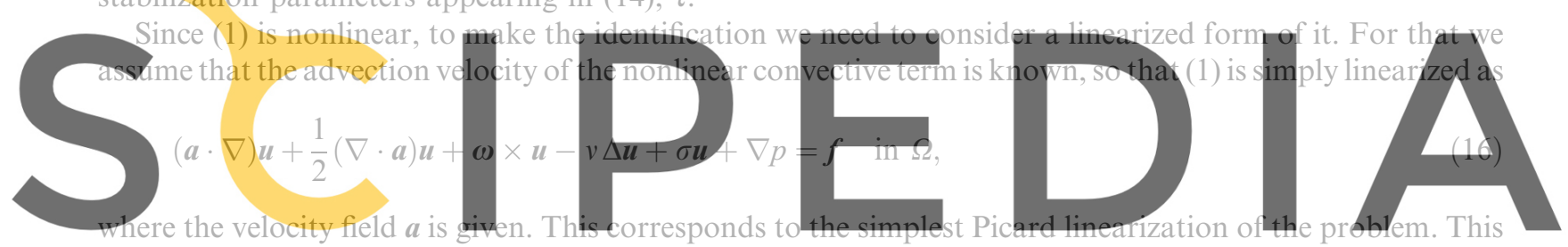

point is discussed further later on.

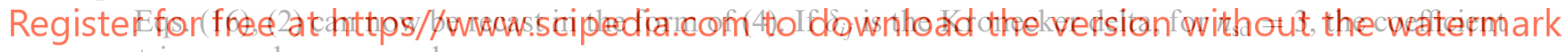
matrices can be expressed as

$$
\begin{aligned}
& \boldsymbol{K}_{i i}=\left[\begin{array}{cccc}
v & 0 & 0 & 0 \\
0 & v & 0 & 0 \\
0 & 0 & v & 0 \\
0 & 0 & 0 & 0
\end{array}\right], \quad \boldsymbol{A}_{i}=\left[\begin{array}{cccc}
a_{i} & 0 & 0 & \delta_{i 1} \\
0 & a_{i} & 0 & \delta_{i 2} \\
0 & 0 & a_{i} & \delta_{i 3} \\
\delta_{i 1} & \delta_{i 2} & \delta_{i 3} & 0
\end{array}\right], \\
& \boldsymbol{S}=\left[\begin{array}{cccc}
\sigma+\frac{1}{2} \nabla \cdot \boldsymbol{a} & -\omega_{3} & \omega_{2} & 0 \\
\omega_{3} & \sigma+\frac{1}{2} \nabla \cdot \boldsymbol{a} & -\omega_{1} & 0 \\
-\omega_{2} & \omega_{1} & \sigma+\frac{1}{2} \nabla \cdot \boldsymbol{a} & 0 \\
0 & 0 & 0 & \varepsilon
\end{array}\right]
\end{aligned}
$$

for $i=1,2,3$, and $\boldsymbol{K}_{i j}=\mathbf{0}$ for $i \neq j$. Observe that in this case the bilinear form associated to the matrices of the diffusion term is only positive semi-definite. The space where the problem has to be posed is now $\mathscr{W}=\left(H_{0}^{1}(\Omega)\right)^{n_{\mathrm{sd}}} \times \mathscr{Q}$ with

$$
\mathscr{Z}=\left\{q \in L^{2}(\Omega) \mid \int_{\Omega} q \mathrm{~d} \Omega=0\right\} .
$$

We denote by $\mathscr{V}_{h} \subset\left(H_{0}^{1}(\Omega)\right)^{n_{\mathrm{sd}}}$ the velocity finite element space, and by $\mathscr{Q}_{h} \subset \mathscr{Q}$ the pressure finite element space. Both are assumed to be built up using continuous finite element interpolations, possibly of the same order. This only affects the approximation properties of the method, as we shall see. 


\subsection{Stability problems of the Galerkin method}

Let us examine which is the lack of stability of the Galerkin method applied to the problem considered now. Let $\boldsymbol{U}_{h}=\left[u_{1, h}, u_{2, h}, u_{3, h}, p_{h}\right]^{\mathrm{t}}$ and $\boldsymbol{V}_{h}=\left[v_{1, h}, v_{2, h}, v_{3, h}, q_{h}\right]^{\mathrm{t}}$ (for $n_{\mathrm{sd}}=3$ ). The bilinear form associated to the problem given by (6) is now

$$
\begin{aligned}
a\left(\boldsymbol{U}_{h}, \boldsymbol{V}_{h}\right)= & v \int_{\Omega} \nabla \boldsymbol{u}_{h}: \nabla \boldsymbol{v}_{h} \mathrm{~d} \Omega+\int_{\Omega}\left[(\boldsymbol{a} \cdot \nabla) \boldsymbol{u}_{h}+\frac{1}{2}(\nabla \cdot \boldsymbol{a}) \boldsymbol{u}_{h}\right] \cdot \boldsymbol{v}_{h} \mathrm{~d} \Omega+\int_{\Omega}\left(\boldsymbol{\omega} \times \boldsymbol{u}_{h}\right) \cdot \boldsymbol{v}_{h} \mathrm{~d} \Omega \\
& +\int_{\Omega} \sigma \boldsymbol{u}_{h} \cdot \boldsymbol{v}_{h} \mathrm{~d} \Omega+\int_{\Omega} \varepsilon p_{h} q_{h} \mathrm{~d} \Omega-\int_{\Omega} p_{h} \nabla \cdot \boldsymbol{v}_{h} \mathrm{~d} \Omega+\int_{\Omega} q_{h} \nabla \cdot \boldsymbol{u}_{h} \mathrm{~d} \Omega .
\end{aligned}
$$

Taking $\boldsymbol{v}_{h}=\boldsymbol{u}_{h}$ and $p_{h}=q_{h}$ and denoting by $\|\cdot\|$ the $L^{2}$ norm in $\Omega$ we get

$$
a\left(\boldsymbol{U}_{h}, \boldsymbol{U}_{h}\right)=v\left\|\nabla \boldsymbol{u}_{h}\right\|^{2}+\sigma\left\|\boldsymbol{u}_{h}\right\|^{2}+\varepsilon\left\|p_{h}\right\|^{2},
$$

which determines the stability provided by the Galerkin method. It is observed that the pressure, the convective and the Coriolis terms are out of contro1. The stability for the pressure has to be explicitly required by imposing that the finite element spaces to interpolate the velocity and the pressure satisfy the classical inf-sup or Babuška-Brezzi stability condition. To have control on the convective term a sort of streamline diffusion has to be introduced in one way or another. The Coriolis force needs to be controlled by other means (see [2]), since otherwise it may lead to global oscillations in $\Omega$. Finally, if $\sigma$ is large, it is seen from (18) that this will give a good control over the $L^{2}$ norm of the velocity, but this will be at the expense of loosing control over the $H^{1}$ norm of this velocity, which may then show localized oscillations near the boundaries.

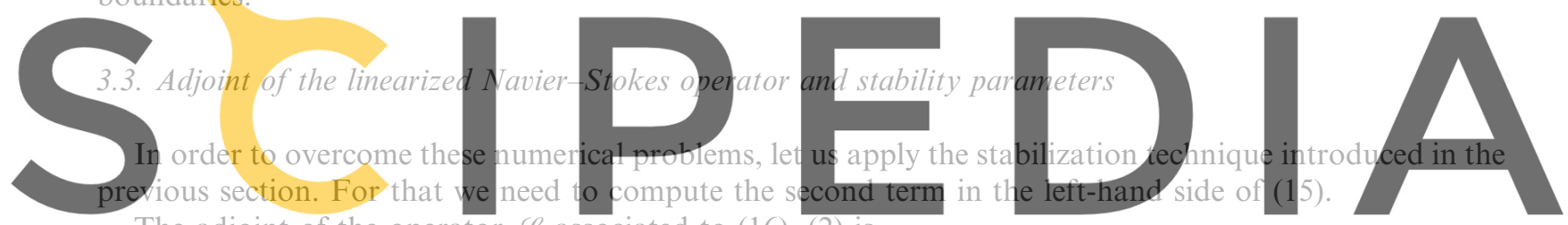

The adjoint of the operator $\mathscr{L}$ associated to (16), (2) is

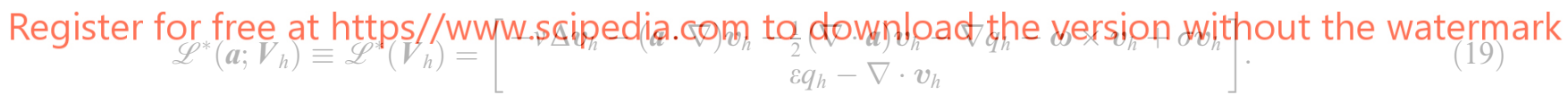

It remains to define the expression for $\tau$. Consider the case $\varepsilon=0$. If we take $\hat{p}=p / \alpha$ and multiply the continuity equation (2) by $\alpha$, the unit coefficients in the $\boldsymbol{A}_{i}$ matrices become $\alpha$, and the velocity does not change. In other words, if the unit coefficients in the $\boldsymbol{A}_{i}$ matrices are multiplied by $\alpha$, the velocity solution is exactly the same. This suggests neglecting these coefficients at the moment of computing $\tau$ and taking it as block diagonal, of the form

$$
\tau=\operatorname{diag}\left(\tau_{1}, \tau_{2}\right),
$$

where $\tau_{1}$ is an $n_{\mathrm{sd}} \times n_{\text {sd }}$ matrix corresponding to the momentum equations. These are coupled through the pressure and the Coriolis force, but the former does not need to appear in $\tau_{1}$. Although the latter produces a coupling between all the momentum equations, we shall see (from the numerical analysis and the numerical results) that the same contribution to $\tau_{1}$ can be used for all the equations. This amounts to saying that matrix $\tau_{1}$ can in fact be taken as $\tau_{1}=\tau_{1} \boldsymbol{I}$, where $\boldsymbol{I}$ is the $n_{\mathrm{sd}} \times n_{\mathrm{sd}}$ identity matrix. Therefore, for $n_{\mathrm{sd}}=3$ the matrix $\tau$ that we shall use is simply

$$
\tau=\operatorname{diag}\left(\tau_{1}, \tau_{1}, \tau_{1}, \tau_{2}\right) .
$$

Remark 1. The argument of the scaling of the pressure is not valid when $\varepsilon>0$. The formulation presented in what follows will not be useful to deal with instabilities arising from large values of $\varepsilon$, and only small 
values of this parameter will be allowed (these are determined in the following). A possible way to deal with the most general problem of large $\varepsilon$ (and even with other terms in the 'continuity' equation) is to use expressions of $\tau$ more general than just a diagonal matrix as in (21).

From expression (21) and from (19) it follows that the terms to be added to the bilinear form of the Galerkin method when the stabilized method is used are

$$
\begin{aligned}
-\int_{\Omega^{\prime}} \mathscr{L}^{*}\left(\boldsymbol{V}_{h}\right)^{\mathrm{t}} \tau \mathscr{L}\left(\boldsymbol{U}_{h}\right) \mathrm{d} \Omega= & \int_{\Omega^{\prime}} \tau_{1}\left[v \Delta \boldsymbol{v}_{h}+(\boldsymbol{a} \cdot \nabla) \boldsymbol{v}_{h}+\frac{1}{2}(\nabla \cdot \boldsymbol{a}) \boldsymbol{v}_{h}+\boldsymbol{\omega} \times \boldsymbol{v}_{h}-\sigma \boldsymbol{v}_{h}+\nabla q_{h}\right] \\
& \cdot\left[-v \Delta \boldsymbol{u}_{h}+(\boldsymbol{a} \cdot \nabla) \boldsymbol{u}_{h}+\frac{1}{2}(\nabla \cdot \boldsymbol{a}) \boldsymbol{u}_{h}+\boldsymbol{\omega} \times \boldsymbol{u}_{h}+\sigma \boldsymbol{u}_{h}+\nabla p_{h}\right] \mathrm{d} \Omega \\
& +\int_{\Omega^{\prime}} \tau_{2}\left(-\varepsilon q_{h}+\nabla \cdot \boldsymbol{v}_{h}\right)\left(\varepsilon p_{h}+\nabla \cdot \boldsymbol{u}_{h}\right) \mathrm{d} \Omega .
\end{aligned}
$$

Now we need to give an expression for $\tau_{1}$ and $\tau_{2}$ in terms of the coefficients of the operator $\mathscr{L}$. Observe first of all that $\tau_{1}$ must have dimensions of time and $\tau_{2}$ of (kinematic) viscosity.

From the analysis of a 1D convection-diffusion-reaction problem presented in [13] based on the discrete maximum principle and, above all, from the numerical analysis of the following section, it turns out that $\tau_{1}$ may be taken within each element as

$$
\tau_{1}=\left(\frac{c_{1} v}{h^{2}}+\frac{c_{2}|\boldsymbol{a}|_{\infty}}{h}+c_{3}|\omega|+\sigma\right)^{-1},
$$

where $c_{1}, c_{2}$ and $c_{3}$ are constants independent of the operator coefficients and the element size $h$ and $|a|$

the supremum of the Euclidea

before (recall that $\varepsilon$ is assun

wilk be clear from the conver

Renark 2. If instead of being a scalar

all what follows would be valid using

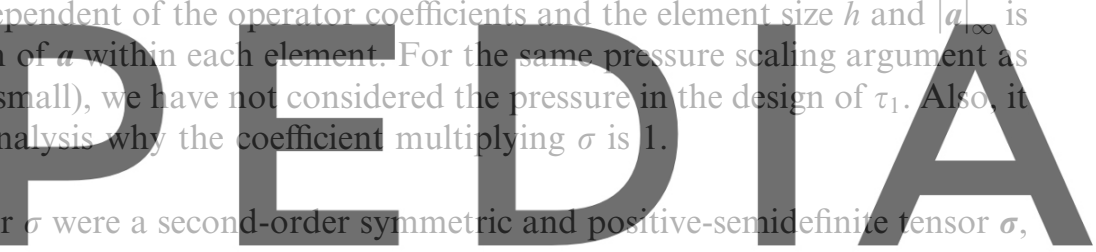

Register for free at httpss//www.scipedia.com to download the version without the watermark $\tau_{1}=\left[\left(\frac{1}{h^{2}}+\frac{c_{1} \infty}{h}+c_{3}|\omega|\right) I+\sigma\right]$

and (20) for $\tau$.

Remark 3. It is important to note that though both $\sigma$ and $\frac{1}{2}(\nabla \cdot \boldsymbol{a})$ contribute to the diagonal of $\boldsymbol{S}$ in (17), they play a different role in expression (23). The latter is in fact considered as part of the convective operator, together with $(\boldsymbol{a} \cdot \nabla)$, and both contribute to $\tau_{1}$ with the term multiplied by $c_{2}$.

Concerning the parameter $\tau_{2}$, it helps to improve the control on the divergence of the velocity and it is found to be effective in practice. Its design is exclusively dictated by the convergence analysis of the following section. We take it as

$$
\tau_{2}=c_{4} v+c_{5}|\boldsymbol{a}|_{\infty} h+c_{6}|\boldsymbol{\omega}| h^{2},
$$

where $c_{4}, c_{5}$ and $c_{6}$ are additional numerical constants.

It will be useful in the following to define

$$
\varphi_{1}:=\frac{c_{1} v}{h^{2}}+\frac{c_{2}|\boldsymbol{a}|_{\infty}}{h}+c_{3}|\boldsymbol{\omega}|
$$

and $\varphi_{2}$ as the inverse of $\tau_{2}$, so that the parameters $\tau_{1}$ and $\tau_{2}$ can be written as

$$
\tau_{1}=\left(\varphi_{1}+\sigma\right)^{-1}, \quad \tau_{2}=\varphi_{2}^{-1} .
$$


Remark 4. Although it is not the purpose of this paper to analyze what happens when $\varepsilon$ is large, let us mention that the use of $\tau_{2}$ given by (24) in this case may lead to an ill-posed problem. This can be circumvented by using

$$
\tau_{2}^{\varepsilon}=\left(\varphi_{2}+\varepsilon\right)^{-1}
$$

instead of $\tau_{2}$. This leads to a method that is well posed an optimally convergent in all the cases except when $\sigma$ is large. We shall come back to this point later on.

Concerning the choice of the constants appearing in (23) and (24), we take $c_{1}=4, c_{2}=2$ and $c_{3}=1$ for linear elements, a choice justified from the analysis of the 1D convection-diffusion-reaction equation and from many numerical experiments. These numerical experiments are less conclusive for the choice of $c_{4}, c_{5}$ and $c_{6}$. Taking them as $c_{4}=4, c_{5}=2$ and $c_{6}=1$ turns out to be effective. For the numerical analysis carried out in Section 4 we shall need that $\varphi_{1}^{-1}$ behaves as $h^{2} \varphi_{2}$. More precisely, there must be two positive constants $c$ and $c^{\prime}$ such that

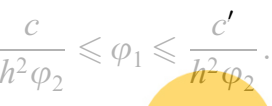

For quadratic elements we use the same values of the algorithmic constants but taking $h$ half the element size (roughly the distance between nodes of the element).

Remark 5. For $c_{4}=1, c_{5}=c_{6}=0$ and $\varepsilon=0$, it is seen from (24) that the contribution of the term multiplied by $\tau_{2}$ is
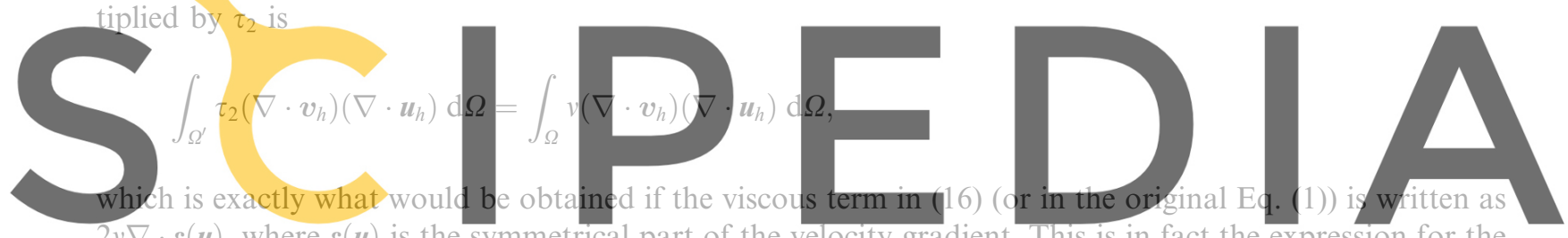
viscous term coming from the conservation of momentum without making use of the continuity equation.

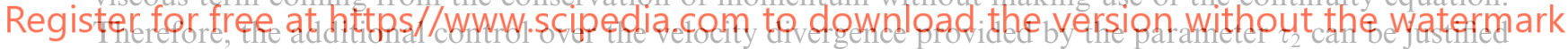

on physical grounds. It is a (kinematic) numerical viscosity that provides control over the volumetric part of the strain rate.

\subsection{Stabilized method for the nonlinear problem}

Let us consider now the original nonlinear equations (1), (2). To apply the previous stabilization method is in principle straightforward. The idea is to add the term (22) to the Galerkin variational formulation of the problem replacing $\boldsymbol{a}$ by $\boldsymbol{u}$, both as advection velocity and in expression (23) to compute $\tau_{1}$. In this case, the stability analysis of the following section carries over to the nonlinear case.

Using a superscript for the iteration counter, let us approximate the convective term at the $i$ th iteration as

$$
\begin{aligned}
{\left[\left(\boldsymbol{u}_{h} \cdot \nabla\right) \boldsymbol{u}_{h}+\frac{1}{2}\left(\nabla \cdot \boldsymbol{u}_{h}\right) \boldsymbol{u}_{h}\right]^{i} \approx } & \left(\boldsymbol{u}_{h}^{i-1} \cdot \nabla\right) \boldsymbol{u}_{h}^{i}+\frac{1}{2}\left(\nabla \cdot \boldsymbol{u}_{h}^{i-1}\right) \boldsymbol{u}_{h}^{i}+\beta\left(\boldsymbol{u}_{h}^{i} \cdot \nabla\right) \boldsymbol{u}_{h}^{i-1}+\beta \frac{1}{2}\left(\nabla \cdot \boldsymbol{u}_{h}^{i}\right) \boldsymbol{u}_{h}^{i-1} \\
& -\beta\left(\boldsymbol{u}_{h}^{i-1} \cdot \nabla\right) \boldsymbol{u}_{h}^{i-1}-\beta \frac{1}{2}\left(\nabla \cdot \boldsymbol{u}_{h}^{i-1}\right) \boldsymbol{u}_{h}^{i-1}
\end{aligned}
$$

where $\beta=0$ for the Picard method and $\beta=1$ for the Newton-Raphson linearization. If the momentum equations were weighted by a test function independent of the velocity field, it is known that Picard's method would yield a linear convergence rate and Newton-Raphson's method a quadratic one (provided the initial guess is close enough to the final solution). However, in our case the momentum equation will be multiplied by $\tau_{1}\left[v \Delta \boldsymbol{v}_{h}+\boldsymbol{u}_{h} \cdot \nabla \boldsymbol{v}_{h}+\boldsymbol{\omega} \times \boldsymbol{v}_{h}-\sigma \boldsymbol{v}_{h}+\nabla q_{h}\right]$, with $\tau_{1}$ depending also on $\boldsymbol{u}_{h}$. Therefore, the 
nonlinearity of the fully discrete problem is not only quadratic, but in fact very complex due to the dependence of $\tau_{1}$ on $\boldsymbol{u}_{h}$.

In our calculations we have evaluated $\tau_{1}$ and $-\mathscr{L}^{*}\left(\boldsymbol{u}_{h} ; \boldsymbol{V}_{h}\right)$ using the velocity of the previous iteration $\left(\boldsymbol{u}_{h}^{i-1}\right.$, with the previous notation), and the convective term multiplied by $-\mathscr{L}^{*}\left(\boldsymbol{u}_{h} ; \boldsymbol{V}_{h}\right)$ approximated by $\left(\boldsymbol{u}_{h}^{i-1} \cdot \nabla\right) \boldsymbol{u}_{h}^{i}$. However, we have left open the possibility of using (28) even with $\beta=1$ for the approximation of the convective term coming from the Galerkin contribution. Even though only a linear convergence rate can be expected in the most general situation, sometimes better convergence is found by taking $\beta=1$. Of course this depends on the relative importance of the stabilization term over the term coming from the Galerkin method, which will be the only one linearized with a second-order scheme.

Having this consideration in mind, we can already write down the iterative scheme for the nonlinear Navier-Stokes equations using the stabilized finite element method presented in this paper. This is detailed in Box 1. Iterations of this scheme are required until convergence is achieved. In all the examples presented in Section 5 we have used a relative tolerance of $0.01 \%$ in the discrete $L^{2}$ norm. The initial guess in all the cases has been set to $\boldsymbol{u}_{h}^{0}=\mathbf{0}$, so that in fact the first iteration corresponds to a Stokes problem. These numerical experiments indicate that this stabilized finite element formulation yields stable and accurate results. Moreover, the particular design of the stabilization matrix presented herein allows to obtain simple stability and error estimates for the linear problem. This is what is shown in Section 4, where, in particular, the role of $\tau_{2}$ in the error analysis becomes apparent.

\section{Box 1. Iterative scheme for the nonlinear problem}

\section{Given a guess $\boldsymbol{u}_{h}^{i-1} \in \mathscr{V}_{h}$, find $\left(\boldsymbol{u}_{h}^{i}, p_{h}^{i}\right) \in \mathscr{V}_{h} \times \mathscr{Q}_{h}=\mathscr{W}_{h}$ such that}
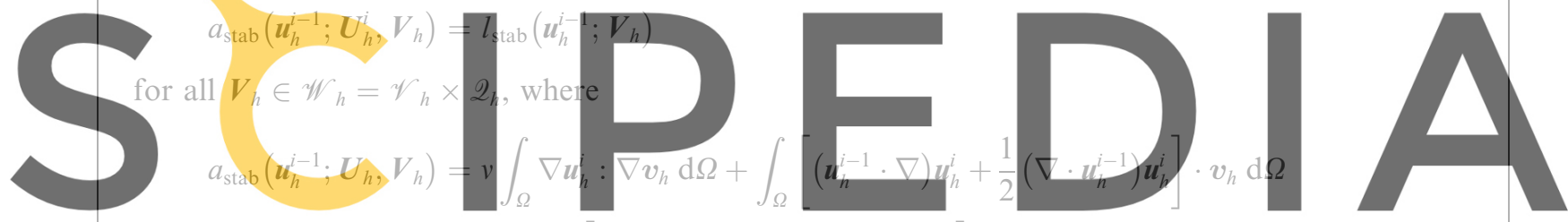

Register for free at https//www.soipedia.com to download the version without the watermark

$$
\begin{aligned}
& +\int_{\Omega} \sigma \boldsymbol{u}_{h}^{i} \cdot v_{h} \mathrm{~d} \Omega+\int_{\Omega} \varepsilon p_{h}^{i} q_{h} \mathrm{~d} \Omega-\int_{\Omega} p_{h}^{i} \nabla \cdot v_{h} \mathrm{~d} \Omega+\int_{\Omega} q_{h} \nabla \cdot u_{h}^{i} \mathrm{~d} \Omega \\
& +\int_{\Omega^{\prime}} \tau_{1}^{i-1}\left[v \Delta \boldsymbol{v}_{h}+\left(\boldsymbol{u}_{h}^{i-1} \cdot \nabla\right) \boldsymbol{v}_{h}+\boldsymbol{\omega} \times \boldsymbol{v}_{h}-\sigma \boldsymbol{v}_{h}+\nabla q_{h}\right] \\
& \cdot\left[-v \Delta \boldsymbol{u}_{h}^{i}+\left(\boldsymbol{u}_{h}^{i-1} \cdot \nabla\right) \boldsymbol{u}_{h}^{i}+\boldsymbol{\omega} \times \boldsymbol{u}_{h}^{i}+\sigma \boldsymbol{u}_{h}^{i}+\nabla p_{h}^{i}\right] \mathrm{d} \Omega \\
& +\int_{\Omega^{\prime}} \tau_{2}^{i-1}\left(-\varepsilon q_{h}+\nabla \cdot \boldsymbol{v}_{h}\right)\left(\varepsilon p_{h}^{i}+\nabla \cdot \boldsymbol{u}_{h}^{i}\right) \mathrm{d} \Omega, \\
& l_{\text {stab }}\left(\boldsymbol{u}_{h}^{i-1} ; \boldsymbol{V}_{h}\right)=\int_{\Omega} \boldsymbol{v}_{h} \cdot \boldsymbol{f} \mathrm{d} \Omega+\beta \int_{\Omega}\left[\left(\boldsymbol{u}_{h}^{i-1} \cdot \nabla\right) \boldsymbol{u}_{h}^{i-1}+\frac{1}{2}\left(\nabla \cdot \boldsymbol{u}_{h}^{i-1}\right) \boldsymbol{u}_{h}^{i-1}\right] \cdot \boldsymbol{v}_{h} \mathrm{~d} \Omega \\
& +\int_{\Omega^{\prime}} \tau_{1}^{i-1}\left[v \Delta \boldsymbol{v}_{h}+\left(\boldsymbol{u}_{h}^{i-1} \cdot \nabla\right) \boldsymbol{v}_{h}+\boldsymbol{\omega} \times \boldsymbol{v}_{h}-\sigma \boldsymbol{v}_{h}+\nabla q_{h}\right] \cdot \boldsymbol{f} \mathrm{d} \Omega,
\end{aligned}
$$

and where the parameters $\tau_{1}$ and $\tau_{2}$ are evaluated within each element as

$$
\begin{aligned}
\tau_{1}^{i-1} & =\left(\frac{c_{1} v}{h^{2}}+\frac{c_{2}\left|\boldsymbol{u}_{h}^{i-1}\right|_{\infty}}{h}+c_{3}|\omega|+\sigma\right)^{-1}, \\
\tau_{2}^{i-1} & =c_{4} v+c_{5}\left|\boldsymbol{u}_{h}^{i-1}\right|_{\infty} h+c_{6}|\boldsymbol{\omega}| h^{2} .
\end{aligned}
$$




\section{Analysis of the linearized problem}

\subsection{Preliminaries}

In this section, we study the stability and convergence of the stabilized finite element method introduced before for the linearized Navier-Stokes equations. The problem to be solved is one of the iterations of the scheme in Box 1 dropping the dependence of $a_{\text {stab }}$ and $l_{\text {stab }}$ on the iteration and setting $\beta=0, \boldsymbol{u}_{h}^{i-1} \equiv \boldsymbol{a}$.

Let us introduce now some notation for what follows. The $L^{2}$ product in a domain $D$ (for scalars, vectors or tensors) is denoted by $(\cdot, \cdot)_{D}$ and the associated $L^{2}$ norm by $\|\cdot\|_{D}$. Subscript $D$ is omitted when $D=\Omega$. The norm in a space $X$ of functions defined on $D$ is denoted by $\|\cdot\|_{X(D)}$ (we use this notation for $X(D)=H^{m}(D)$ and $X(D)=L^{\infty}(D)$ ).

For finite element functions $f_{h}$ and $g_{h}$ and for a set of parameters $\tau$ defined within each element, we write:

$$
\left(f_{h}, \tau g_{h}\right)_{h}:=\sum_{e=1}^{n_{\mathrm{el}}} \int_{\Omega^{e}} f_{h} \tau g_{h} \mathrm{~d} \Omega, \quad\left\|\tau^{1 / 2} f_{h}\right\|_{h}:=\sqrt{\left(f_{h}, \tau f_{h}\right)_{h}} .
$$

To simplify the notation, we use the abbreviations

$$
X\left(v_{h}, q_{h}\right):=(a \cdot \nabla) v_{h}+\frac{1}{2}(\nabla \cdot a) v_{h}+\omega \times v_{h}+\nabla q_{h},
$$

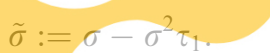

By virtue of expression (23) for $\tau_{1}, \tilde{\sigma}$ is nonnegative. Moreover, from (25) it is seen that it can be written as
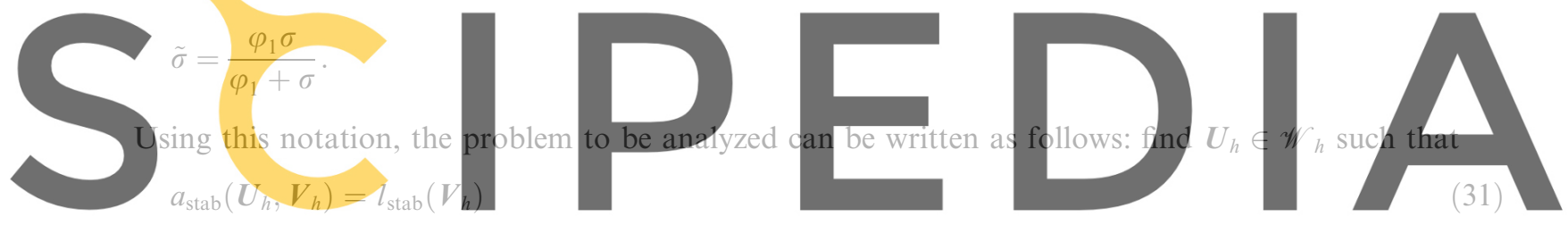

for all $V_{h} \in \mathscr{W}_{h}$, where

Register for free at https/www.scipedia.com to download the version without the watermark

$$
\begin{aligned}
&+\left(v \Delta v_{h}+X\left(v_{h}, q_{h}\right)-\sigma v_{h}, \tau_{1}\left(-v \Delta u_{h}+X\left(\boldsymbol{w}_{h}, p_{h}\right)+\sigma \boldsymbol{u}_{h}\right)\right)_{h} \\
&+\left(-\varepsilon q_{h}+\nabla \cdot \boldsymbol{v}_{h}, \tau_{2}\left(\varepsilon p_{h}+\nabla \cdot \boldsymbol{u}_{h}\right)\right)_{h}, \\
& l_{\text {stab }}\left(\boldsymbol{V}_{h}\right)=\left(\boldsymbol{v}_{h}, \boldsymbol{f}\right)+\left(v \Delta \boldsymbol{v}_{h}+X\left(\boldsymbol{v}_{h}, q_{h}\right)-\sigma \boldsymbol{v}_{h}, \tau_{1} \boldsymbol{f}\right)_{h} .
\end{aligned}
$$

We assume that $f \in\left(L^{2}(\Omega)\right)^{n_{\mathrm{sd}}}$ in (33) and in the following.

As it has been mentioned, the parameter $\varepsilon$ must be small. The precise condition that we shall need is

$$
\varepsilon \leqslant \frac{C_{1}}{h^{2}\left(\varphi_{1}+\sigma\right)} \leqslant C_{2} \varphi_{2}, \quad 0 \leqslant C_{1}, \quad 0 \leqslant C_{2}<1
$$

The stability and convergence of this method will be proven in the norm ||$\cdot \mid \|$, defined as

$$
\left\|\boldsymbol{V}_{h}\right\| \mid:=\left[v\left\|\nabla \boldsymbol{v}_{h}\right\|^{2}+\left\|\tilde{\sigma}^{1 / 2} \boldsymbol{v}_{h}\right\|_{h}^{2}+\varepsilon\left\|q_{h}\right\|^{2}+\left\|\tau_{1}^{1 / 2} X\left(\boldsymbol{v}_{h}, q_{h}\right)\right\|_{h}^{2}+\left\|\tau_{2}^{1 / 2} \nabla \cdot \boldsymbol{v}_{h}\right\|_{h}^{2}\right]^{1 / 2} .
$$

The implications of using this norm are deferred to the end of this section.

Under the assumption that the family of finite element meshes obtained from successive refinements is nondegenerate, the following inverse estimates hold (see, e.g., [19]):

$$
\left\|\Delta v_{h}\right\|_{\Omega^{e}} \leqslant \frac{C_{\mathrm{inv}}}{h^{e}}\left\|\nabla v_{h}\right\|_{\Omega^{e}}
$$




$$
\begin{aligned}
& \left\|\nabla v_{h}\right\|_{\Omega^{e}} \leqslant \frac{C_{\mathrm{inv}}}{h^{e}}\left\|v_{h}\right\|_{\Omega^{e}}, \\
& \left\|v_{h}\right\|_{L^{\infty}\left(\Omega^{e}\right)} \leqslant \frac{C_{\text {inv }}}{\left(h^{e}\right)^{n_{\mathrm{sd}} / 2}}\left\|v_{h}\right\|_{\Omega^{e}}
\end{aligned}
$$

for $e=1, \ldots, n_{\mathrm{el}}$, where $C_{\mathrm{inv}}$ is a constant, $v_{h}$ a finite element function, belonging either to the velocity or to the pressure space, and $\|\cdot\|_{L^{\infty}\left(\Omega^{e}\right)}$ is the supremum norm.

The final ingredient we need is a mesh regularity condition in the case $\sigma>0$. Let $\Gamma^{b}$ be an interior boundary of the finite element partition, i.e., a face in $3 \mathrm{D}$ or edge in $2 \mathrm{D}$ shared by two elements, say $e_{1}$ and $e_{2}$. Let also $\Omega^{b}$ be the domain obtained from the union of the domains of elements $e_{1}$ and $e_{2}$, i.e.,

$$
\overline{\Omega^{b}}=\overline{\Omega^{e_{1}} \cup \Omega^{e_{2}}}
$$

the overbar denoting the closure of a domain. The number of such boundaries will be called $n_{\mathrm{bo}}$ in the following. The condition we need is that the jump of $\varphi_{1}$ at $\Gamma^{b}$ be bounded as the mesh size goes to zero, which can be expressed by saying that there are positive constants $c$ and $c^{\prime}$ such that

$$
c \varphi_{1, e_{1}} \leqslant \varphi_{1, e_{2}} \leqslant c^{\prime} \varphi_{1, e_{1}} .
$$

This condition means in particular that the ratio of the sizes between two adjacent elements is bounded as the mesh is refined, which is a consequence of the fact that the family of finite element meshes is nondegenerate.

From now on, $C$ will denote a generic positive constant, independent of the mesh size, possibly different at different occurrences.

\subsection{Stability and convergence}

We are now in a position to prove stability and convergence for problem (31). We will proceed in a very classical way, first proving stability, then continuity of $a_{\text {stab }}\left(\boldsymbol{U}_{h}, \boldsymbol{V}_{h}\right)$ in a different norm for $\boldsymbol{U}_{h}$ and $\boldsymbol{V}_{h}$ and finally we will prove convergence using a Céa-type argument.

Lemma 1. Assume that $\tau_{1}$ is given by (23) and the constant $c_{1}$ is $c_{1}>\alpha C_{\mathrm{inv}}^{2}$, with $\alpha>1$ and $C_{\mathrm{inv}}$ the constant in the inverse estimates (36), (37). Then there exists a positive constant $C$ such that

$$
a_{\text {stab }}\left(\boldsymbol{U}_{h}, \boldsymbol{U}_{h}\right) \geqslant C \mid\left\|\boldsymbol{U}_{h}\right\|^{2}
$$

for all $\boldsymbol{U}_{h} \in \mathscr{W}_{h}$.

Proof. Taking $\boldsymbol{V}_{h}=\boldsymbol{U}_{h}$ in (32) it is found that

$$
\begin{aligned}
a_{\text {stab }}\left(\boldsymbol{U}_{h}, \boldsymbol{U}_{h}\right)= & v\left\|\nabla \boldsymbol{u}_{h}\right\|^{2}+\sigma\left\|\boldsymbol{u}_{h}\right\|^{2}+\varepsilon\left\|p_{h}\right\|^{2}-\left\|\tau_{1}^{1 / 2}\left(v \Delta \boldsymbol{u}_{h}-\sigma \boldsymbol{u}_{h}\right)\right\|_{h}^{2}+\left\|\tau_{1}^{1 / 2} X\left(\boldsymbol{u}_{h}, p_{h}\right)\right\|_{h}^{2} \\
& +\left\|\tau_{2}^{1 / 2} \nabla \cdot \boldsymbol{u}_{h}\right\|_{h}^{2}-\varepsilon^{2}\left\|\tau_{2}^{1 / 2} p_{h}\right\|_{h}^{2} \\
\geqslant & v\left\|\nabla \boldsymbol{u}_{h}\right\|^{2}+\sigma\left\|\boldsymbol{u}_{h}\right\|^{2}+\varepsilon\left\|p_{h}\right\|^{2}-v^{2}\left\|\tau_{1}^{1 / 2} \Delta \boldsymbol{u}_{h}\right\|_{h}^{2}-\sigma^{2}\left\|\tau_{1}^{1 / 2} \boldsymbol{u}_{h}\right\|_{h}^{2}-2 v \sigma\left\|\tau_{1}^{1 / 2} \Delta \boldsymbol{u}_{h}\right\|\left\|_{h}\right\| \tau_{1}^{1 / 2} \boldsymbol{u}_{h} \|_{h} \\
& +\left\|\tau_{1}^{1 / 2} X\left(\boldsymbol{u}_{h}, p_{h}\right)\right\|_{h}^{2}+\left\|\tau_{2}^{1 / 2} \nabla \cdot \boldsymbol{u}_{h}\right\|_{h}^{2}-\varepsilon^{2}\left\|\tau_{2}^{1 / 2} p_{h}\right\|_{h}^{2} \cdot
\end{aligned}
$$


Using now the inverse estimates (36), (37) and the fact that for any $x$ and $y$ and for any $\alpha>0$

$$
-2 x y \geqslant-\frac{1}{\alpha} x^{2}-\alpha y^{2}
$$

it follows that

$$
\begin{gathered}
a_{\text {stab }}\left(\boldsymbol{U}_{h}, \boldsymbol{U}_{h}\right) \geqslant v\left\|\nabla \boldsymbol{u}_{h}\right\|^{2}+\sigma\left\|\boldsymbol{u}_{h}\right\|^{2}+\varepsilon\left\|p_{h}\right\|^{2}-v^{2} C_{\mathrm{inv}}^{2}\left\|\tau_{1}^{1 / 2} \frac{1}{h} \nabla \boldsymbol{u}_{h}\right\|_{h}^{2}-\sigma^{2}\left\|\tau_{1}^{1 / 2} \boldsymbol{u}_{h}\right\|_{h}^{2} \\
\quad-v \sigma\left(\frac{1}{\alpha}\left\|\tau_{1}^{1 / 2} \nabla \boldsymbol{u}_{h}\right\|_{h}^{2}+C_{\mathrm{inv}}^{2} \alpha\left\|\tau_{1}^{1 / 2} \frac{1}{h} \boldsymbol{u}_{h}\right\|_{h}^{2}\right)+\left\|\tau_{1}^{1 / 2} X\left(\boldsymbol{u}_{h}, p_{h}\right)\right\|_{h}^{2}+\left\|\tau_{2}^{1 / 2} \nabla \cdot \boldsymbol{u}_{h}\right\|_{h}^{2}-\varepsilon^{2}\left\|\tau_{2}^{1 / 2} p_{h}\right\|_{h}^{2} \\
=\sum_{e=1}^{n_{\mathrm{cl}}}\left\{\tau_{1}\left(\frac{v}{\tau_{1}}-v^{2} C_{\mathrm{inv}}^{2} \frac{1}{h^{2}}-\frac{1}{\alpha} v \sigma\right)\left\|\nabla \boldsymbol{u}_{h}\right\|_{\Omega^{e}}^{2}+\tau_{1}\left(\frac{\sigma}{\tau_{1}}-\sigma^{2}-\alpha \sigma v C_{\mathrm{inv}}^{2} \frac{1}{h^{2}}\right)\left\|\boldsymbol{u}_{h}\right\|_{\Omega^{e}}^{2}\right. \\
\left.\quad+\varepsilon\left(1-\varepsilon \tau_{2}\right)\left\|p_{h}\right\|_{\Omega^{e}}^{2}+\tau_{1}\left\|X\left(\boldsymbol{u}_{h}, p_{h}\right)\right\|_{\Omega^{e}}^{2}+\tau_{2}\left\|\nabla \cdot \boldsymbol{u}_{h}\right\|_{\Omega^{e}}^{2}\right\}
\end{gathered}
$$

From condition (34) we have that the coefficient multiplying the norm of $p_{h}$ within each element is

$$
\varepsilon\left(1-\varepsilon \tau_{2}\right) \geqslant C \varepsilon
$$

On the other hand, from expression (23) for $\tau_{1}$ and the assumption on the constant $c_{1}$, it follows that, for $\alpha>1$, the coefficient of $\left\|\nabla \boldsymbol{u}_{h}\right\|_{\Omega^{e}}^{2}$ is

$$
v \tau_{1}\left[\frac{v}{h^{2}}\left(c_{1}-C_{\mathrm{inv}}^{2}\right)+\frac{c_{2}|\boldsymbol{a}|_{\infty}}{h}+c_{3}|\boldsymbol{\omega}|+\left(1-\frac{1}{\alpha}\right) \sigma\right] \geqslant C v,
$$

and the coefficient of $\left\|\boldsymbol{u}_{h}\right\|_{\Omega^{e}}^{2}$ is

$$
\sigma \tau_{1}\left[\frac{v}{h^{2}}\left(c_{1}-\alpha C_{\text {inv }}^{2}\right)+\frac{c_{2}|\boldsymbol{a}|_{\infty}}{h}+c_{3}|\omega|\right] \geqslant C \tilde{\sigma}
$$

Observe that to obtain this inequality it is essential that the coefficient multiplying $\sigma$ in (23) be 1 . The stability estimate (40) follows from the last three inequalities applied to (41).

From estimate (40) it is observed that now we have control on $X\left(\boldsymbol{u}_{h}, p_{h}\right)$ (the term 'unseen' by the Galerkin method) as well as on $\nabla \cdot \boldsymbol{u}_{h}$. Observe that to arrive to (40) we have made use of the expression of $\tau_{1}$, but not of $\tau_{2}$. However, we need both in order to prove a certain continuity of $a_{\text {stab. }}$.

Lemma 2. Assume that $\tau_{1}$ and $\tau_{2}$ are given by (23) and (24), respectively, with $c_{1}$ and the rest of algorithmic constants strictly positive, so that condition (27) is satisfied. Then there exists a positive constant $C$ such that

$$
a_{\text {stab }}\left(\boldsymbol{U}_{h}, \boldsymbol{V}_{h}\right) \leqslant C\left[\left\|\frac{\tau_{2}^{1 / 2}}{h} \boldsymbol{u}_{h}\right\|_{h}+\left\|\frac{\tau_{1}^{1 / 2}}{h} p_{h}\right\|_{h}\right]\left\|\boldsymbol{V}_{h}\right\| \|
$$

for all $\boldsymbol{U}_{h}, \boldsymbol{V}_{h} \in \mathscr{W}_{h}$. 
Proof. From (32) and using only Schwartz's inequality for some terms we have that

$$
\begin{aligned}
a_{\text {stab }}\left(\boldsymbol{U}_{h}, \boldsymbol{V}_{h}\right) \leqslant & v\left\|\nabla \boldsymbol{u}_{h}\right\|,\left\|\nabla \boldsymbol{v}_{h}\right\| \\
& +\left(\boldsymbol{v}_{h}, X\left(\boldsymbol{u}_{h}, p_{h}\right)\right)_{h} \\
& +\sigma\left(\boldsymbol{u}_{h}, \boldsymbol{v}_{h}\right) \\
& +\left(q_{h}, \nabla \cdot \boldsymbol{u}_{h}\right)_{h} \\
& +\varepsilon\left(p_{h}, q_{h}\right) \\
& +v^{2}\left\|\tau_{1} \Delta \boldsymbol{u}_{h}\right\|_{h}\left\|\Delta \boldsymbol{v}_{h}\right\|_{h} \\
& +v\left\|\tau_{1} \Delta \boldsymbol{u}_{h}\right\|_{h}\left\|X\left(\boldsymbol{v}_{h}, q_{h}\right)\right\|_{h} \\
& +v \sigma\left\|\tau_{1} \Delta \boldsymbol{u}_{h}\right\|_{h}\left\|\boldsymbol{v}_{h}\right\|_{h} \\
& +v\left\|\tau_{1} X\left(\boldsymbol{u}_{h}, p_{h}\right)\right\|_{h}\left\|\Delta \boldsymbol{v}_{h}\right\|_{h} \\
& +\left\|\tau_{1} X\left(\boldsymbol{u}_{h}, p_{h}\right)\right\|_{h}\left\|X\left(\boldsymbol{v}_{h}, q_{h}\right)\right\|_{h} \\
& -\sigma\left(\tau_{1} X\left(\boldsymbol{u}_{h}, p_{h}\right), \boldsymbol{v}_{h}\right)_{h} \\
& +\sigma v\left\|\tau_{1} \boldsymbol{u}_{h}\right\|_{h}\left\|\Delta \boldsymbol{v}_{h}\right\|_{h} \\
& +\sigma\left(\tau_{1} \boldsymbol{u}_{h}, X\left(\boldsymbol{v}_{h}, q_{h}\right)\right)_{h} \\
& -\sigma^{2}\left(\tau_{1} \boldsymbol{u}_{h}, \boldsymbol{v}_{h}\right)_{h} \\
& -\varepsilon^{2}\left(\tau_{2} p_{h}, q_{h}\right)_{h} \\
& +\varepsilon\left(\nabla \cdot \boldsymbol{v}_{h}, \tau_{2} p_{h}\right)_{h} \\
& -\varepsilon\left(\tau_{2} \nabla \cdot \boldsymbol{u}_{h}, q_{h}\right)_{h} \\
& +\left\|\tau_{2} \nabla \cdot \boldsymbol{u}_{h}\right\|_{h}\left\|\nabla \cdot \boldsymbol{v}_{h}\right\| .
\end{aligned}
$$

Now we have to bound these 18 terms using the norm (35) of $\boldsymbol{V}_{h}$ and a certain norm of $\boldsymbol{U}_{h}$. There are some of these terms that offer no difficulty. These are (42), (51) and (59), since their addition can be written as

$$
v\left\|\nabla \boldsymbol{u}_{h}\right\|\left\|\nabla \boldsymbol{v}_{h}\right\|+\left\|\tau_{1}^{1 / 2} X\left(\boldsymbol{u}_{h}, p_{h}\right)\right\|_{h}\left\|\tau_{1}^{1 / 2} X\left(\boldsymbol{v}_{h}, q_{h}\right)\right\|_{h}+\left\|\tau_{2}^{1 / 2} \nabla \cdot \boldsymbol{u}_{h}\right\|_{h}\left\|\tau_{2}^{1 / 2} \nabla \cdot \boldsymbol{v}_{h}\right\|_{h} \leqslant C\left|\left\|\boldsymbol{U}_{h}|||||| \boldsymbol{V}_{h} \mid\right\| .\right.
$$

Terms (44) and (55) can be grouped together to yield

$$
\sigma\left(\boldsymbol{u}_{h}, \boldsymbol{v}_{h}\right)-\sigma^{2}\left(\boldsymbol{u}_{h}, \tau_{1} \boldsymbol{v}_{h}\right)_{h}=\left(\boldsymbol{u}_{h}, \tilde{\sigma} \boldsymbol{v}_{h}\right)_{h} \leqslant C||\left|\boldsymbol{U}_{h}\right||||| \boldsymbol{V}_{h}|| \mid,
$$

and, similarly, for terms (46) and (56) we have, using condition (34), that

$$
\varepsilon\left(p_{h}, q_{h}\right)-\varepsilon^{2}\left(p_{h}, \tau_{2} q_{h}\right)_{h} \leqslant 2 \varepsilon\left\|p_{h}\right\|\left\|q_{h}\right\| \leqslant C\left|\left\|\boldsymbol{U}_{h}\right\|\right|\left\|\mid \boldsymbol{V}_{h}\right\| \| .
$$

Terms (47)-(49) can be bounded using the inverse estimates (36), (37) and expression (23) for $\tau_{1}$ :

$$
\begin{aligned}
& v^{2}\left\|\tau_{1}^{1 / 2} \Delta \boldsymbol{u}_{h}\right\|\left\|_{h}\right\| \tau_{1}^{1 / 2} \Delta \boldsymbol{v}_{h}\left\|_{h}+v\right\| \tau_{1}^{1 / 2} \Delta \boldsymbol{u}_{h}\left\|_{h}\right\| \tau_{1}^{1 / 2} X\left(\boldsymbol{v}_{h}, q_{h}\right)\left\|_{h}+v \sigma\right\| \tau_{1}^{1 / 2} \Delta \boldsymbol{u}_{h}\left\|_{h}\right\| \tau_{1}^{1 / 2} \boldsymbol{v}_{h} \|_{h} \\
& \leqslant v\left\|v^{1 / 2} C_{\text {inv }} \frac{1}{h} \tau_{1}^{1 / 2} \nabla \boldsymbol{u}_{h}\right\|\left\|v_{h}^{1 / 2} C_{\text {inv }} \frac{1}{h} \tau_{1}^{1 / 2} \nabla \boldsymbol{v}_{h}\right\|_{h} \\
& \quad+v^{1 / 2}\left\|v^{1 / 2} C_{\text {inv }} \frac{1}{h} \tau_{1}^{1 / 2} \nabla \boldsymbol{u}_{h}\right\|\left\|\tau_{h}^{1 / 2} X\left(\boldsymbol{v}_{h}, q_{h}\right)\right\|_{h}+\sigma\left\|v C_{\text {inv }}^{2} \frac{1}{h^{2}} \tau_{1} \boldsymbol{u}_{h}\right\|_{h}\left\|\boldsymbol{v}_{h}\right\| \\
& \leqslant v\left\|\nabla \boldsymbol{u}_{h}\right\|\left\|\nabla \boldsymbol{v}_{h}\right\|+v^{1 / 2}\left\|\nabla \boldsymbol{u}_{h}\right\|\left\|\tau_{1}^{1 / 2} X\left(\boldsymbol{v}_{h}, q_{h}\right)\right\|_{h}+\left\|\tilde{\sigma}^{1 / 2} \boldsymbol{u}_{h}\right\|_{h}\left\|\tilde{\sigma}^{1 / 2} \boldsymbol{v}_{h}\right\|_{h} \\
& \leqslant C\left\|\boldsymbol{U}_{h}\right\| \mid\left\|\boldsymbol{V}_{h}\right\| .
\end{aligned}
$$

Term (50) can be bounded as (48) and (53) as (49), so that

$$
v\left\|\tau_{1}^{1 / 2} X\left(\boldsymbol{u}_{h}, p_{h}\right)\right\|_{h}\left\|\tau_{1}^{1 / 2} \Delta \boldsymbol{v}_{h}\right\|_{h}+\sigma v\left\|\tau_{1}^{1 / 2} \boldsymbol{u}_{h}\right\|_{h}\left\|\tau_{1}^{1 / 2} \Delta \boldsymbol{v}_{h}\right\|_{h} \leqslant C\left|\left\|\boldsymbol { U } _ { h } \left|\left\|\left|\left\|\mid \boldsymbol{V}_{h}\right\| \|\right.\right.\right.\right.\right.
$$


Let us bound now (54). Using definition (29) of $X\left(\boldsymbol{v}_{h}, q_{h}\right)$ we have

$$
\begin{aligned}
\sigma\left(\tau_{1} \boldsymbol{u}_{h}, X\left(\boldsymbol{v}_{h}, q_{h}\right)\right)_{h} & \leqslant \sigma\left\|\boldsymbol{u}_{h}\right\|\left\|\tau_{1}\left(\frac{C_{\mathrm{inv}}|\boldsymbol{a}|_{\infty}}{h}+|\boldsymbol{\omega}|\right) \boldsymbol{v}_{h}\right\|_{h}+\sigma\left(\tau_{1} \boldsymbol{u}_{h}, \nabla q_{h}\right)_{h} \\
& \leqslant C\left\|\tilde{\sigma}^{1 / 2} \boldsymbol{u}_{h}\right\|\left\|_{h}\right\| \tilde{\sigma}^{1 / 2} \boldsymbol{v}_{h} \|_{h}+\sigma\left(\tau_{1} \boldsymbol{u}_{h}, \nabla q_{h}\right)_{h} \\
& \leqslant C\|\| \boldsymbol{U}_{h}\|\|\left\|\left|\boldsymbol{V}_{h}\right|\right\|+\sigma\left(\tau_{1} \boldsymbol{u}_{h}, \nabla q_{h}\right)_{h} .
\end{aligned}
$$

Adding now the second term in the right-hand side with (43), (45) and (52) and integrating by parts within each element the convective term we have

$$
\begin{aligned}
& \sigma\left(\tau_{1} \boldsymbol{u}_{h}, \nabla q_{h}\right)_{h}-\sigma\left(\tau_{1} X\left(\boldsymbol{u}_{h}, p_{h}\right), \boldsymbol{v}_{h}\right)_{h}+\left(q_{h}, \nabla \cdot \boldsymbol{u}_{h}\right)+\left(\boldsymbol{v}_{h}, X\left(\boldsymbol{u}_{h}, p_{h}\right)\right) \\
&=-\sigma\left(\tau_{1} \boldsymbol{v}_{h}, \nabla p_{h}\right)_{h}+\sigma\left(\tau_{1} X\left(\boldsymbol{v}_{h}, q_{h}\right), \boldsymbol{u}_{h}\right)_{h}+\sum_{e=1}^{n_{\mathrm{el}}} \int_{\partial \Omega^{e}} \sigma \tau_{1}(\boldsymbol{n} \cdot \boldsymbol{a})\left(\boldsymbol{u}_{h} \cdot \boldsymbol{v}_{h}\right) \mathrm{d} \Gamma \\
& \quad-\left(p_{h}, \nabla \cdot \boldsymbol{v}_{h}\right)-\left(\boldsymbol{u}_{h}, X\left(\boldsymbol{v}_{h}, q_{h}\right)\right) \\
&=:[\mathrm{I}]+[\mathrm{II}]+[\mathrm{III}]+[\mathrm{IV}]+[\mathrm{V}] .
\end{aligned}
$$

We proceed now to bound each of these five terms. For $[\mathrm{I}]+[\mathrm{IV}]$ we have, integrating again by parts within each element,

$$
\begin{aligned}
\sigma\left(\tau_{1} \boldsymbol{v}_{h}, \nabla p_{h}\right)_{h}+\left(p_{h}, \nabla \cdot \boldsymbol{v}_{h}\right) & =\left(\left(1-\sigma \tau_{1}\right) p_{h}, \nabla \cdot \boldsymbol{v}_{h}\right)_{h}+\sum_{e=1}^{n_{\mathrm{el}}} \int_{\partial \Omega^{e}} \sigma \tau_{1} \boldsymbol{n} \cdot \boldsymbol{v}_{h} p_{h} \mathrm{~d} \Gamma, \\
& =\left(\frac{\varphi_{1}}{\varphi_{1}+\sigma} p_{h}, \nabla \cdot \boldsymbol{v}_{h}\right)_{h}+\sum_{b=1}^{n_{\mathrm{bo}}} \int_{\Gamma^{b}} \sigma \llbracket \tau_{1} \rrbracket \boldsymbol{n} \cdot \boldsymbol{v}_{h} p_{h} \mathrm{~d} \Gamma,
\end{aligned}
$$

where $\llbracket \tau_{1} \rrbracket$ is the jump of $\tau_{1}$ across the interior boundary $\Gamma^{b}$ and we have made use of the continuity of the velocity and pressure finite element functions across this boundary. The normal appearing in (67) is the exterior to $\Omega^{e}$ for the integral over $\partial \Omega^{e}$ and a fixed normal to $\Gamma^{b}$ for the integral over this boundary. Using relationship (27), the first term in (67) can be bounded as follows:

$$
\begin{aligned}
\left(\frac{\varphi_{1}}{\varphi_{1}+\sigma} p_{h}, \nabla \cdot \boldsymbol{v}_{h}\right)_{h} & \leqslant\left\|\varphi_{1}^{1 / 2} h \nabla \cdot \boldsymbol{v}_{h}\right\|_{h}\left\|\frac{1}{\left(\varphi_{1}+\sigma\right)^{1 / 2} h} p_{h}\right\|_{h} \\
& \leqslant C\left\|\tau_{2}^{1 / 2} \nabla \cdot \boldsymbol{v}_{h}\right\|_{h}\left\|\frac{\tau_{1}^{1 / 2}}{h} p_{h}\right\|_{h} \\
& \leqslant C\|\| \boldsymbol{V}_{h}\|\| \frac{\tau_{1}^{1 / 2}}{h} p_{h} \|_{h}
\end{aligned}
$$

The second term in (67) can be bounded making use of the inverse estimate (38) and condition (39). If $e_{1}$ and $e_{2}$ are the two elements sharing $\Gamma^{b}$, the measure of which is denoted meas $\left(\Gamma^{b}\right)$, we have

$$
\begin{aligned}
& \sum_{b=1}^{n_{\mathrm{bo}}} \int_{\Gamma^{b}} \sigma \llbracket \tau_{1} \rrbracket \boldsymbol{n} \cdot \boldsymbol{v}_{h} p_{h} \mathrm{~d} \Gamma \\
& \quad \leqslant \sum_{b=1}^{n_{\mathrm{bo}}} \sigma \frac{\left|\varphi_{1, e_{1}}-\varphi_{1, e_{2}}\right|}{\left(\varphi_{1, e_{1}}+\sigma\right)\left(\varphi_{1, e_{2}}+\sigma\right)}\left\|\boldsymbol{v}_{h}\right\|_{L^{\infty}\left(\Omega^{b}\right)}\left\|p_{h}\right\|_{L^{\infty}\left(\Omega^{b}\right)} \operatorname{meas}\left(\Gamma^{b}\right) \\
& \quad \leqslant C \sum_{b=1}^{n_{\mathrm{bo}}} \sum_{i=1}^{2} \frac{\sigma \varphi_{1, e_{i}}}{\left(\varphi_{1, e_{i}}+\sigma\right)^{2}} \frac{C_{\mathrm{inv}}^{2}}{\left(h^{e_{i}}\right)^{n_{\mathrm{sd}}}}\left\|\boldsymbol{v}_{h}\right\|_{\Omega^{e_{i}}}\left\|p_{h}\right\|_{\Omega^{e_{i}}}\left(h^{e_{i}}\right)^{n_{\mathrm{sd}}-1}
\end{aligned}
$$




$$
\begin{aligned}
& \leqslant C \sum_{e=1}^{n_{\mathrm{el}}} \frac{\sigma^{1 / 2} \varphi_{1}^{1 / 2}}{\left(\varphi_{1}+\sigma\right)^{1 / 2}} \frac{1}{\left(\varphi_{1}+\sigma\right)^{1 / 2}} \frac{1}{h}\left\|\boldsymbol{v}_{h}\right\|_{\Omega^{e}}\left\|p_{h}\right\|_{\Omega^{e}} \\
& \leqslant C\left\|\tilde{\sigma}^{1 / 2} \boldsymbol{v}_{h}\right\|\left\|\frac{\tau_{1}^{1 / 2}}{h} p_{h}\right\|_{h} \\
& \leqslant C\|\| \boldsymbol{V}_{h}\|\| \frac{\tau_{1}^{1 / 2}}{h} p_{h} \|_{h}
\end{aligned}
$$

Let us consider now $[\mathrm{II}]+[\mathrm{V}]$ in (66). Using again (27) we have

$$
\begin{aligned}
& \left(\boldsymbol{u}_{h}, X\left(\boldsymbol{v}_{h}, q_{h}\right)\right)_{h}-\sigma\left(\tau_{1} X\left(\boldsymbol{v}_{h}, q_{h}\right), \boldsymbol{u}_{h}\right)_{h} \\
& \quad=\left(\frac{\varphi_{1}}{\varphi_{1}+\sigma} \boldsymbol{u}_{h}, X\left(\boldsymbol{v}_{h}, q_{h}\right)\right)_{h} \leqslant\left\|\varphi_{1}^{1 / 2} \boldsymbol{u}_{h}\right\|_{h}\left\|\frac{1}{\left(\varphi_{1}+\sigma\right)^{1 / 2}} X\left(\boldsymbol{v}_{h}, q_{h}\right)\right\|_{h} \leqslant C\|\| \boldsymbol{V}_{h}\|\| \frac{\tau_{2}^{1 / 2}}{h} \boldsymbol{u}_{h} \|_{h} .
\end{aligned}
$$

It only remains to bound [III] in (66), for which we can use steps similar to those in (69) and the expression of $\varphi_{1}$ to give

$$
\begin{aligned}
\sum_{e=1}^{n_{\mathrm{el}}} \int_{\partial \Omega^{e}} \sigma \tau_{1}(\boldsymbol{n} \cdot \boldsymbol{a})\left(\boldsymbol{u}_{h} \cdot \boldsymbol{v}_{h}\right) \mathrm{d} \Gamma & =\sum_{b=1}^{n_{\mathrm{bo}}} \int_{\Gamma^{b}} \sigma \llbracket \tau_{1} \rrbracket(\boldsymbol{n} \cdot \boldsymbol{a})\left(\boldsymbol{u}_{h} \cdot \boldsymbol{v}_{h}\right) \mathrm{d} \Gamma \\
& \leqslant C \sum_{e=1}^{n_{\mathrm{el}}} \frac{\sigma \varphi_{1}}{\left(\varphi_{1}+\sigma\right)^{2}} \frac{|\boldsymbol{a}|_{\infty}}{h}\left\|\boldsymbol{u}_{h}\right\|_{\Omega^{e}}\left\|\boldsymbol{v}_{h}\right\|_{\Omega^{e}} \\
& \leqslant C\left\|\tilde{\sigma}^{1 / 2} \boldsymbol{v}_{h}\right\|_{h}\left\|\tilde{\sigma}^{1 / 2} \boldsymbol{u}_{h}\right\|_{h} .
\end{aligned}
$$

Finally, (57) and (58) are easily bounded using condition (34) on the parameter $\varepsilon$ :

$$
\begin{aligned}
\varepsilon\left(\nabla \cdot \boldsymbol{v}_{h}, \tau_{2} p_{h}\right)_{h}-\varepsilon\left(\tau_{2} \nabla \cdot \boldsymbol{u}_{h}, q_{h}\right)_{h} & \leqslant \varepsilon^{1 / 2}\left\|\tau_{2}^{1 / 2} \nabla \cdot \boldsymbol{v}_{h}\right\|_{h}\left\|p_{h}\right\|+\varepsilon^{1 / 2}\left\|\tau_{2}^{1 / 2} \nabla \cdot \boldsymbol{u}_{h}\right\|_{h}\left\|q_{h}\right\| \\
& \leqslant C\left|\left\|\boldsymbol{U}_{h}\right\|\right|\|\mid\| \boldsymbol{V}_{h} \| .
\end{aligned}
$$

Combining (60)-(72) we have that

$$
a_{\text {stab }}\left(\boldsymbol{U}_{h}, \boldsymbol{V}_{h}\right) \leqslant C\left|\left\|\boldsymbol{V}_{h} \mid\right\|\left\{\left\|\boldsymbol{U}_{h}\right\| \mid+\left\|\frac{\tau_{2}^{1 / 2}}{h} \boldsymbol{u}_{h}\right\|_{h}+\left\|\frac{\tau_{1}^{1 / 2}}{h} p_{h}\right\|_{h}\right\} .\right.
$$

It remains to express $\|\left|\boldsymbol{U}_{h}\right|||$ in terms of the other two norms within the braces for a generic finite element function $\boldsymbol{U}_{h}$. It is easy to see that

$$
\begin{aligned}
\left\|\left|\boldsymbol{U}_{h} \|\right|^{2}=\right. & v\left\|\nabla \boldsymbol{u}_{h}\right\|^{2}+\left\|\tilde{\sigma}^{1 / 2} \boldsymbol{u}_{h}\right\|_{h}^{2}+\varepsilon\left\|p_{h}\right\|^{2}+\left\|\tau_{1}^{1 / 2} X\left(\boldsymbol{u}_{h}, p_{h}\right)\right\|_{h}^{2}+\left\|\tau_{2}^{1 / 2} \nabla \cdot \boldsymbol{u}_{h}\right\|_{h}^{2} \\
\leqslant & v\left\|C_{\text {inv }} \frac{1}{h} \boldsymbol{u}_{h}\right\|_{h}^{2}+\left\|\tilde{\sigma}^{1 / 2} \boldsymbol{u}_{h}\right\|_{h}^{2}+\varepsilon\left\|p_{h}\right\|^{2}+3\left\|\tau_{1}^{1 / 2}|\boldsymbol{a}|_{\infty} C_{\text {inv }} \frac{1}{h} \boldsymbol{u}_{h}\right\|_{h}^{2}+3\left\|\tau_{1}^{1 / 2}|\boldsymbol{\omega}| \boldsymbol{u}_{h}\right\|_{h}^{2} \\
& +3\left\|\tau_{1}^{1 / 2} C_{\text {inv }} \frac{1}{h} p_{h}\right\|_{h}^{2}+\left\|\tau_{2}^{1 / 2} C_{\text {inv }} \frac{1}{h} \boldsymbol{u}_{h}\right\|_{h}^{2}
\end{aligned}
$$

The coefficient multiplying the norm of $\boldsymbol{u}_{h}$ within each element is

$$
\begin{aligned}
& C_{\mathrm{inv}} \frac{v^{1 / 2}}{h}+\tilde{\sigma}^{1 / 2}+3 \tau_{1}^{1 / 2}\left(|\boldsymbol{a}|_{\infty} C_{\mathrm{inv}} \frac{1}{h}+|\boldsymbol{\omega}|\right)+\tau_{2}^{1 / 2} C_{\mathrm{inv}} \frac{1}{h} \\
& \leqslant C \varphi_{1}^{1 / 2}\left(1+\frac{\sigma^{1 / 2}}{\left(\varphi_{1}+\sigma\right)^{1 / 2}}+\frac{\varphi_{1}^{1 / 2}}{\left(\varphi_{1}+\sigma\right)^{1 / 2}}\right)+C \frac{\tau_{2}^{1 / 2}}{h} \leqslant C \frac{\tau_{2}^{1 / 2}}{h} .
\end{aligned}
$$


We have used here the expressions of $\tau_{1}$ and $\tau_{2}$ in terms of $\varphi_{1}$ and $\varphi_{2}$, as well as condition (27). Similarly, the coefficient multiplying the norm of $p_{h}$ within each element in (74) is

$$
\varepsilon^{1 / 2}+3 \tau_{1}^{1 / 2} C_{\text {inv }} \frac{1}{h} \leqslant C \frac{\tau_{1}^{1 / 2}}{h},
$$

where we have used condition (34) on the penalty parameter $\varepsilon$. The lemma follows using (75) and (76) in (74) and combining it with (73).

We proceed now to prove convergence. As usual, this can only be proved if the solution of the continuous problem is more regular than actually required for the problem to be well posed. The space where it has to belong is

$$
\mathscr{W}_{r}=\left(H^{m}(\Omega) \cap H_{0}^{1}(\Omega)\right)^{n_{\mathrm{sd}}} \times\left(H^{n}(\Omega) \cap \mathscr{Q}\right),
$$

with $m \geqslant k_{\mathrm{u}}+1$ and $n \geqslant k_{\mathrm{p}}$ and $k_{\mathrm{u}} \geqslant 1, k_{\mathrm{p}} \geqslant 1$ being the order of the finite element interpolation for the velocity and the pressure, respectively.

Let $\hat{\boldsymbol{U}}_{h} \in \mathscr{W}_{h}$ be an interpolant of the solution $\boldsymbol{U}$ of the continuous problem (5), with components $\hat{\boldsymbol{u}}_{h}$ and $\hat{p}_{h}$ corresponding to the velocity and pressure interpolants, respectively. If we define

$$
E_{\text {int }}^{e}(v):=\left(h^{e}\right)^{k_{\mathrm{v}}+1}\|v\|_{H^{k \mathrm{v}+1}\left(\Omega^{e}\right)},
$$

for a continuous function $v$ whose finite element interpolant of order $k_{\mathrm{v}}$ is $\hat{v}_{h}$, the interpolation error within each element satisfies

$$
\left\|v-\hat{v}_{h}\right\|_{H^{m}\left(\Omega^{e}\right)} \leqslant \frac{C}{\left(h^{e}\right)^{m}} E_{\mathrm{int}}^{e}(v) .
$$

We shall apply this for $m=0,1$ for the pressure $(v=p)$ and for $m=0,1,2$ for the velocity $(v=\boldsymbol{u})$. Likewise, when $\sigma>0$ we also assume that the velocity components and the pressure are in $L^{\infty}(\Omega)$. In this case, we have that

$$
\left\|v-\hat{v}_{h}\right\|_{L^{\infty}\left(\Omega^{e}\right)} \leqslant \frac{C}{\left(h^{e}\right)^{n_{\mathrm{sd}} / 2}} E_{\mathrm{int}}^{e}(v) .
$$

Theorem 1. Let $\boldsymbol{U}$ be the solution of the continuous problem, assumed to be $\boldsymbol{U} \in \mathscr{W}_{r}$, with $\mathscr{W}_{r}$ defined in (77). Under the assumptions of Lemmas 1 and 2 on the stabilization parameters, there exists a constant $C$ such that

$$
\left\|\boldsymbol{E}_{h} \mid\right\| \leqslant C \sum_{e=1}^{n_{\mathrm{el}}}\left[\frac{\left(\tau_{2}^{e}\right)^{1 / 2}}{h^{e}} E_{\mathrm{int}}^{e}(\boldsymbol{u})+\frac{\left(\tau_{1}^{e}\right)^{1 / 2}}{h^{e}} E_{\mathrm{int}}^{e}(p)\right],
$$

where $\boldsymbol{E}_{h}=\boldsymbol{U}-\boldsymbol{U}_{h}$.

Proof. If $\boldsymbol{U}$ is the solution of the continuous problem and it is smooth enough (as required in the theorem) it verifies (31), and thus we have the consistency property

$$
a_{\text {stab }}\left(\boldsymbol{U}-\boldsymbol{U}_{h}, \boldsymbol{V}_{h}\right)=0 \quad \forall \boldsymbol{V}_{h} \in \mathscr{W}_{h} .
$$

From this and Lemma 1 we have that

$$
C\left|\left\|\hat{\boldsymbol{U}}_{h}-\boldsymbol{U}_{h} \mid\right\|^{2} \leqslant a_{\text {stab }}\left(\hat{\boldsymbol{U}}_{h}-\boldsymbol{U}_{h}, \hat{\boldsymbol{U}}_{h}-\boldsymbol{U}_{h}\right)=a_{\mathrm{stab}}\left(\hat{\boldsymbol{U}}_{h}-\boldsymbol{U}, \hat{\boldsymbol{U}}_{h}-\boldsymbol{U}_{h}\right) .\right.
$$

Let $\psi(h)$ be the right-hand side of (80). In Lemma 2, which is valid for all finite element functions $\boldsymbol{U}_{h}$ and $\boldsymbol{V}_{h}$, the only properties needed to obtain the norms of $\boldsymbol{u}_{h}$ and $p_{h}$ within the brackets are the inverse estimates (36), (37), and (38) when $\sigma>0$. These can be replaced by the interpolation estimates (78), (79) to show that 


$$
a_{\text {stab }}\left(\hat{\boldsymbol{U}}_{h}-\boldsymbol{U}, \hat{\boldsymbol{U}}_{h}-\boldsymbol{U}_{h}\right) \leqslant C \psi(h)|| \mid \hat{\boldsymbol{U}}_{h}-\boldsymbol{U}_{h}\|\|,
$$

which combined with (81) implies that

$$
\left\|\hat{\boldsymbol{U}}_{h}-\boldsymbol{U}_{h}\right\| \mid \leqslant C \psi(h) .
$$

The theorem follows from this and the triangle inequality:

$$
||\left|\boldsymbol{U}-\boldsymbol{U}_{h}\right||| \leqslant||\left|\boldsymbol{U}-\hat{\boldsymbol{U}}_{h}\right|||+|| \hat{\boldsymbol{U}}_{h}-\boldsymbol{U}_{h}|| \mid \leqslant C \psi(h)+C \psi(h),
$$

where the bound from the first term is obtained as (74) using again (78) instead of the inverse estimates.

Remark 6. In the case in which $\varepsilon$ is large, the previous error estimate does not hold. First of all, $\tau_{2}$ has to be replaced by $\tau_{2}^{\varepsilon}$ given by (26). Also, the norm given by (35) is not the appropriate one to work with. It has to be replaced by

$$
\left\|\mid \boldsymbol{V}_{h}\right\| \|_{\varepsilon}:=\left[v\left\|\nabla \boldsymbol{v}_{h}\right\|^{2}+\left\|\tilde{\sigma}^{1 / 2} \boldsymbol{v}_{h}\right\|_{h}^{2}+\left\|\tilde{\varepsilon}^{1 / 2} q_{h}\right\|_{h}^{2}+\left\|\tau_{1}^{1 / 2} X\left(\boldsymbol{v}_{h}, q_{h}\right)\right\|_{h}^{2}+\left\|\tau_{2}^{1 / 2} \nabla \cdot \boldsymbol{v}_{h}\right\|_{h}^{2}\right]^{1 / 2}
$$

where

$$
\tilde{\varepsilon}^{1 / 2}:=\varepsilon\left(1-\tau_{2}^{\varepsilon} \varepsilon\right)=\frac{\varepsilon \varphi_{2}}{\left(\varepsilon+\varphi_{2}\right)},
$$

and $\tau_{2}^{\varepsilon}$ is given by (26). We give without proof the following result:

Theorem 2. Under the same assumptions as in Theorem 1 , if $\tau_{2}^{\varepsilon}$ is used instead of $\tau_{2}$, there exists a constant $C$ such that

$$
\left\|\boldsymbol{E}_{h} \mid\right\|_{\varepsilon} \leqslant C \sum_{e=1}^{n_{\mathrm{el}}}\left[\left(\varphi_{1}^{e}\right)^{1 / 2} E_{\mathrm{int}}^{e}(\boldsymbol{u})+\left(\varphi_{2}^{e}\right)^{1 / 2} E_{\mathrm{int}}^{e}(p)\right] .
$$

\subsection{Discussion}

The purpose of this section is to apply the error estimate (80) to the limiting cases found when only one of the physical parameters $v,|\boldsymbol{a}|_{\infty},|\boldsymbol{\omega}|$ or $\sigma$ is dominant. This will show why this error estimate can be considered as 'optimal'. For each case, the contribution of the terms that do not dominate will be neglected.

In order to simplify the discussion, we consider here that the finite element mesh is quasi-uniform and of diameter $h$, so that $(80)$ reduces to

$$
\left\|\boldsymbol{E}_{h}\right\| \mid \leqslant C\left[\tau_{2}^{1 / 2} h^{k_{\mathrm{u}}}+\tau_{1}^{1 / 2} h^{k_{\mathrm{p}}}\right]
$$

Recall that $k_{\mathrm{u}}$ and $k_{\mathrm{p}}$ are the orders of the polynomial approximation for the velocity and the pressure, respectively.

Case 1. $v$ dominant: In this case, problem (31) is the standard Stokes problem. After some rearrangements, the error estimate (82) can be written as

$$
\left\|\nabla \boldsymbol{e}_{\mathrm{u}}\right\|+\frac{h}{v}\left\|\nabla e_{\mathrm{p}}\right\|+\left\|\nabla \cdot \boldsymbol{e}_{\mathrm{u}}\right\| \leqslant C\left[h^{k_{\mathrm{u}}}+\frac{1}{v} h^{k_{\mathrm{p}}+1}\right] .
$$

Here and in the following, $\boldsymbol{e}_{\mathrm{u}}:=\boldsymbol{u}-\boldsymbol{u}_{h}$ and $e_{\mathrm{p}}:=p-p_{h}$ are the velocity and pressure finite element errors, respectively. It is seen that these finite element errors are of the same order as the interpolation errors, and therefore optimal (observe that the factor $1 / v$ can be included in the pressure). 
Even though in (83) the pressure error is expressed in terms of the gradient of $e_{\mathrm{p}}$, it is possible to obtain a pressure error estimate in $L^{2}$. This can be done either by relying on the inf-sup condition satisfied by the continuous problem (as done in [20]) or by using duality arguments (the approach followed in [21]).

From (83) it is seen that if $k_{\mathrm{p}}+1=k_{\mathrm{u}}$, then both terms on the right-hand side are of the same order, a situation that could be considered optimal. However, this is not true for the cases discussed next.

Case 2. $|\boldsymbol{a}|_{\infty}$ dominant: When $|\boldsymbol{a}|_{\infty}$ dominates, the error estimate (82) can be written as

$$
\frac{1}{|\boldsymbol{a}|_{\infty}}\left\|(\boldsymbol{a} \cdot \nabla) \boldsymbol{e}_{\mathrm{u}}+\nabla e_{\mathrm{p}}\right\|+\left\|\nabla \cdot \boldsymbol{e}_{\mathrm{u}}\right\| \leqslant C\left[h^{k_{\mathrm{u}}}+\frac{1}{|\boldsymbol{a}|_{\infty}} h^{k_{\mathrm{p}}}\right],
$$

which is optimal. In this case, it is convenient to scale the pressure by the factor $1 /|\boldsymbol{a}|_{\infty}$. The additional control obtained with respect to the Galerkin method is the error estimate for the derivative along the streamline-direction as well as the error estimate for the velocity divergence.

Case 3. $|\omega|$ dominant: This case is similar to the previous one. When $|\omega|$ dominates (82) leads to

$$
\frac{1}{|\boldsymbol{\omega}|}\left\|\boldsymbol{\omega} \times \boldsymbol{e}_{\mathrm{u}}+\nabla e_{\mathrm{p}}\right\|+h\left\|\nabla \cdot \boldsymbol{e}_{\mathrm{u}}\right\| \leqslant C\left[h^{k_{\mathrm{u}}+1}+\frac{1}{|\boldsymbol{\omega}|} h^{k_{\mathrm{p}}}\right],
$$

which is optimal. In this case, it is convenient to scale the pressure by the factor $1 /|\omega|$. As it could be expected from the differential equation to be solved, the optimal orders of approximation for the velocity and pressure finite element spaces in this case would be such that $k_{\mathrm{p}}=k_{\mathrm{u}}+1$.

Case 4. $\sigma$ dominant: From the definition of $\tilde{\sigma}$ in (30) it turns out that when $\sigma \rightarrow \infty$ then $\tilde{\sigma} \rightarrow v / h^{2}$. The error estimate (82) in this case yields

$$
\left\|\nabla \boldsymbol{e}_{\mathrm{u}}\right\|+\frac{1}{h}\left\|\boldsymbol{e}_{\mathrm{u}}\right\|+\frac{1}{(\sigma v)^{1 / 2}}\left\|\nabla e_{\mathrm{p}}\right\| \leqslant C\left[h^{k_{\mathrm{u}}}+\frac{1}{(\sigma v)^{1 / 2}} h^{k_{\mathrm{p}}}\right] .
$$

As in the previous cases, this error estimate is optimal. However, the improvement achieved with respect to the Galerkin method in this case is different from the others. Instead of getting control over a new term of the differential equation, now the improvement comes from the fact that the errors in the velocity gradient and the velocity itself are 'well balanced', as it can be observed from (84). For the Galerkin method, the factor $1 / h$ in this estimate has to be replaced by $(\sigma / v)^{1 / 2}$ and therefore only a $L^{2}$ velocity error estimate can be obtained for very large values of $\sigma$. Velocity derivatives are out of control. In practice, localized oscillations may appear near the boundaries when $\sigma$ dominates over the rest of coefficients of the equation.

If instead of using the result of Theorem 1 we rely on the error estimate of Theorem 2, we obtain

$$
\left\|\nabla \boldsymbol{e}_{\mathrm{u}}\right\|+\frac{1}{h}\left\|\boldsymbol{e}_{\mathrm{u}}\right\|+\frac{1}{(\sigma v)^{1 / 2}}\left\|\nabla e_{\mathrm{p}}\right\| \leqslant C\left[h^{k_{\mathrm{u}}}+\frac{1}{v} h^{k_{\mathrm{p}}+1}\right] .
$$

In contrast to what happens with (84), now it is not possible to scale the pressure by $1 /(\sigma v)^{1 / 2}$. Estimate (85) deteriorates as $\sigma \rightarrow \infty$, loosing control over the pressure.

\section{Numerical examples}

The following numerical examples are intended to show the performance of the stabilized finite element formulation studied in this paper compared to the standard Galerkin approach. Since it is based on the algebraic approximation to the sub-scales given by (14), it is referred to as the ASGS (standing for algebraic sub-grid scale) method.

In the absence of rotation and permeability effects, the ASGS method is very close to the GLS or SUPG methods (they reduce to the same method for linear elements), whose performance in convection dominated problems is well known. Therefore, attention shall be focussed here on the numerical performance when either the $\omega$ or the $\sigma$ (or both) coefficients are large and convection is not important. In all the examples in the following, $\varepsilon=0$. 
When the Galerkin method is used, div-stable velocity-pressure interpolations satisfying the inf-sup condition need to be employed. The pairs used in the following are the $Q_{1} / P_{0}$ (bilinear velocities and piecewise constant pressures, which is not exactly div-stable but is known to yield good results), the $Q_{2} / Q_{1}$ (biquadratic velocities and continuous bilinear pressures) and the $P_{2} / P_{1}$ (quadratic velocities and continuous linear pressures). For the ASGS method equal velocity-pressure can be used, but it is obviously possible to use div-stable interpolations in this case as well.

\subsection{Convergence test}

As a first case, let us consider a 2D steady state test with analytical solution to check the behavior in space of the finite element approximation to problem (1)-(3). This test has also been presented in [22] using a slightly different stabilized method. We take $\Omega$ as the unit square and the force term so that the exact solution is $p=0$ and $\boldsymbol{u}(x, y)=\left(f(x) g^{\prime}(y),-f^{\prime}(x) g(y)\right)$, with $f(x)=x^{2}(1-x)^{2} \exp (7 x)$ and $g(y)=$ $y^{2}(1-y)^{2}$. This velocity field vanishes on $\partial \Omega$.

As physical properties we have taken $v=0.005$ and different values of $\omega$ and $\sigma$. In particular, results will be shown for $\sigma=0,1000$ and $\omega=0,1000$. We have used three uniform finite element meshes (meshes 1,2 and 3 ) of $10 \times 10,20 \times 20$ and $40 \times 40$ bilinear elements, so that the element sizes are $h=0.1, h=0.05$ and $h=0.025$, respectively. The resulting values of the element Reynolds number are not very high and for this particular example the standard Galerkin approach using the $Q_{1} / P_{0}$ element works in the absence of Coriolis force. However, when this force exists, the Galerkin method yields completely oscillatory results all over the computational domain. Results for $\omega=1000$ and $\sigma=0$ are shown in Fig. 1, where also the streamlines obtained using the ASGS method are shown. In this case there are no oscillations.

In Fig. 2 we have plotted the convergence of the velocities obtained with the ASGS method as the mesh is refined, both in the $H^{1}$ and the $L^{2}$ norms and for different combinations of the values of $\sigma$ and $\omega$. The convergence rate is optimal in both norms and for all the combinations of $\sigma$ and $\omega$.

Similar results are obtained in the 3D extension of this example that we consider now. The domain is $\bar{\Omega}=[0,1] \times[0,1] \times[0,0.4]$ and is first discretized using a coarse mesh of $10 \times 10 \times 4$ elements. We take the force term so as to obtain as exact solution $\boldsymbol{u}(x, y, z)=\left(h(z) f(x) g^{\prime}(y),-h(z) f^{\prime}(x) g(y), 0\right)$, with $f(x)$ and $g(y)$

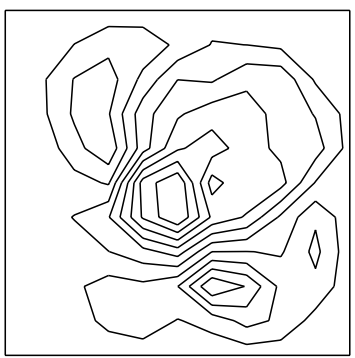

(1)

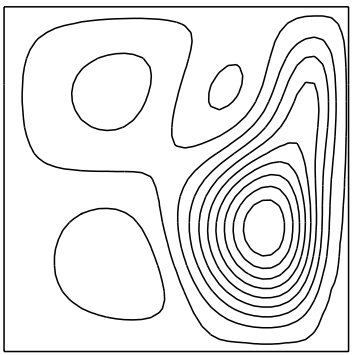

(3)

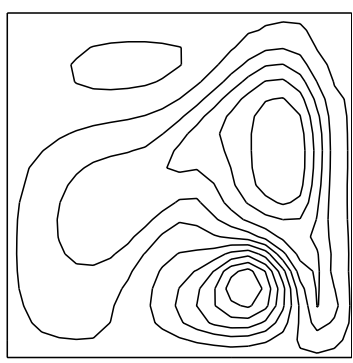

(2)

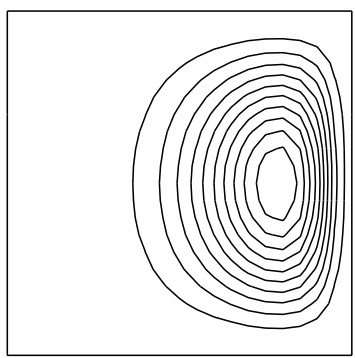

(4)

Fig. 1. (1) Streamlines using the Galerkin method with mesh $1\left(Q_{1} / P_{0}\right.$ element); (2) same as (1) with mesh 2; (3) same as (1) with mesh 3 ; (4) streamlines using the ASGS method with mesh $1\left(Q_{1} / Q_{1}\right.$ element). 

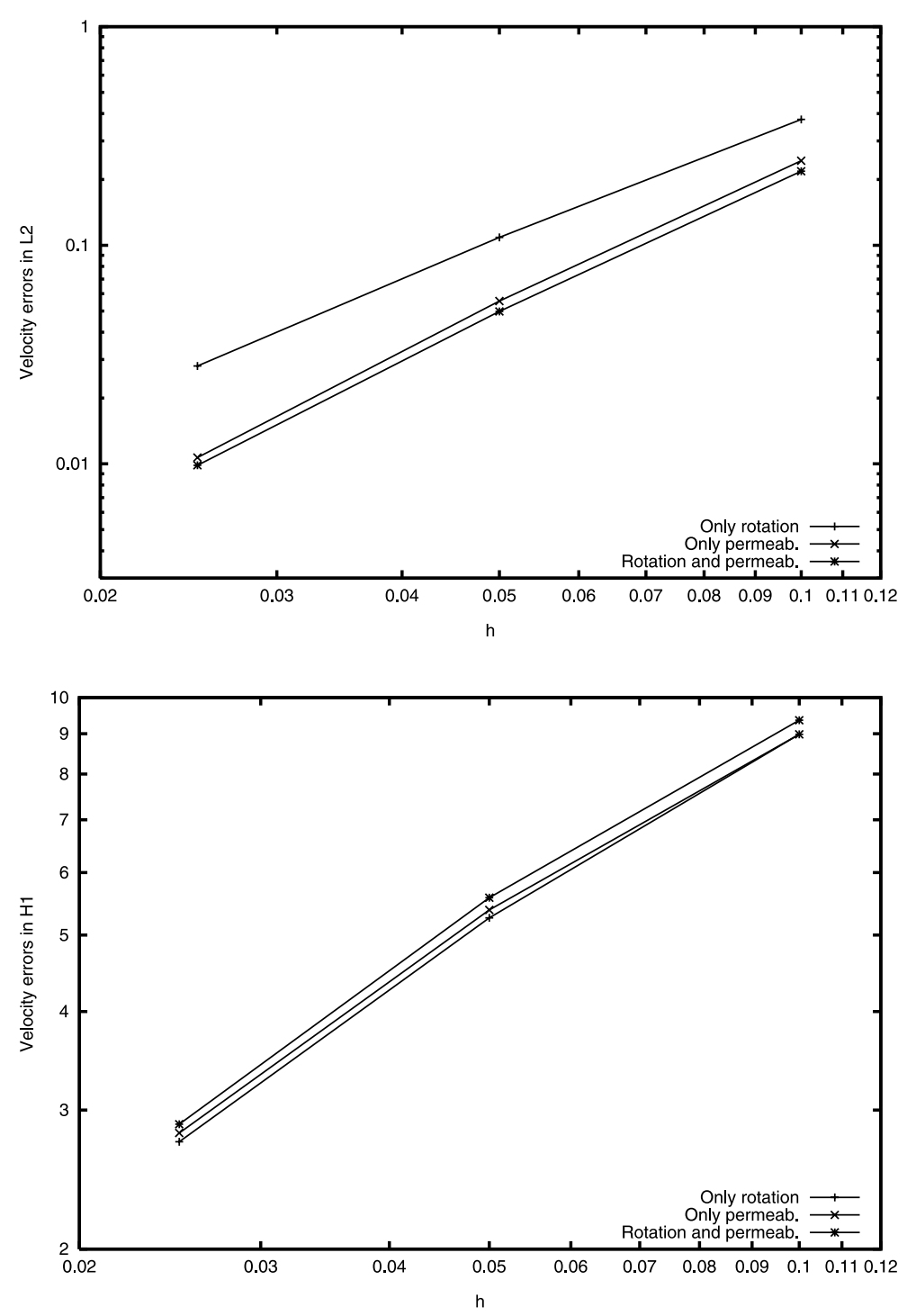

Fig. 2. Velocity convergence of the ASGS method using equal velocity-pressure interpolation. Top: $L^{2}$ norm. Bottom: $H^{1}$ norm.

as before and $h(z)=z(10-25 z)$. In order to test the numerical method, we have taken different vectors $\omega$, all with the same norm $\omega=1000$. In all the cases we have obtained good solutions using the ASGS method.

In Fig. 3 we have plotted the velocity vectors obtained for $\omega$ parallel to $(1,1,1)$ and $\sigma=0$, both for the standard Galerkin method and the ASGS method. The oscillations found using the former are completely removed by the latter.

\subsection{Rotating Poiseuille flow}

In this example (also presented in [22]) we consider a 2D Poiseuille rotating flow with $\sigma=0$. The computational domain is the rectangle $[-2,2] \times[-1,1]$, which rotates about the origin with a speed of rotation $\omega=100$. The Reynolds number is taken small enough so as to neglect the convective term of the Navier-Stokes equations. The problem is therefore linear.

A parabolic velocity profile with maximum velocity $(1,0)$ is prescribed at the inlet $x=-2$, whereas at the top and bottom walls $(y=-1,1)$ the no-slip condition is employed. If the velocity is also prescribed at the outlet $x=2$, the velocity solution would not be affected by the fact the domain rotates (both the Coriolis 

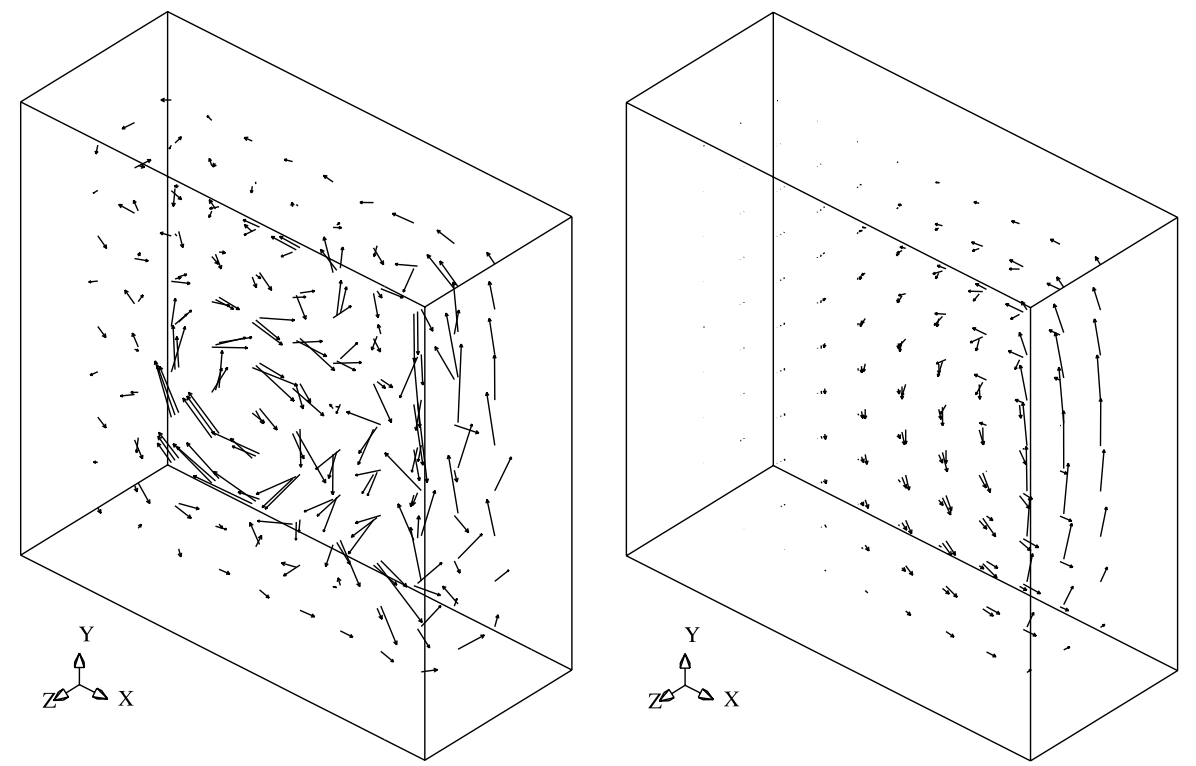

Fig. 3. Velocity vectors for the $3 \mathrm{D}$ test case. Left: Galerkin method ( $Q_{1} / P_{0}$ element). Right: ASGS method ( $Q_{1} / Q_{1}$ element).

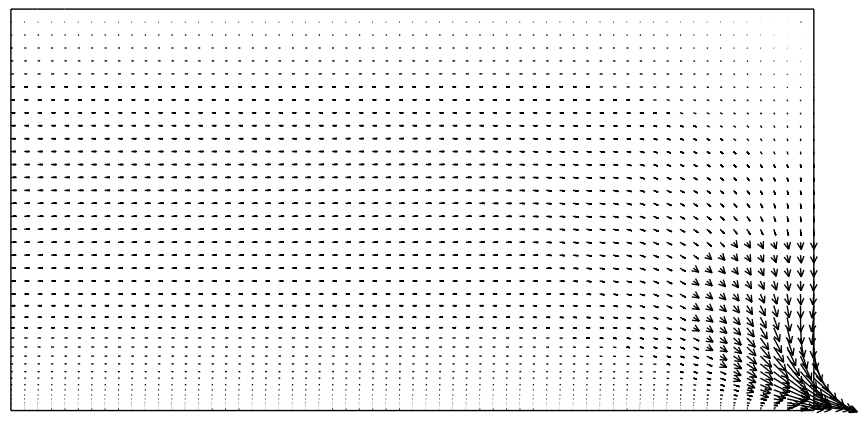

Fig. 4. Velocity field for the rotating Poiseuille flow problem (without centrifugal force). Galerkin method with $v=1$ using the $Q_{2} / Q_{1}$ element.

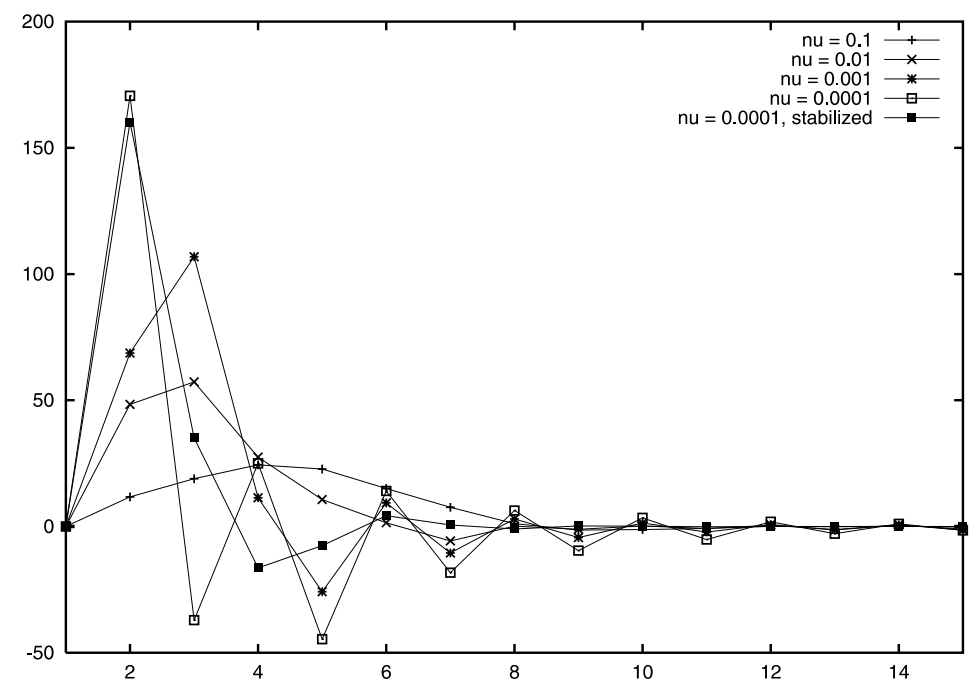

Fig. 5. $x$-Velocity profiles at the outlet for different values of the viscosity using the Galerkin method (with the $Q_{2} / Q_{1}$ element). Results using the ASGS method (with the $Q_{2} / Q_{2}$ element) are only shown for $v=10^{-4}$. Abscissa in grid units. 
and centrifugal forces are curl-free, and therefore they can be written as the gradient of a scalar function that can be included in the pressure). Instead of prescribing the velocity, we have used the homogeneous Neumann boundary condition associated to the Stokes operator, i.e.,

$$
-p \boldsymbol{n}+v \frac{\partial \boldsymbol{u}}{\partial n}=\mathbf{0} \quad \text { at } x=2
$$

It turns out that for this very simple problem a velocity 'boundary layer' is created at the outlet when $\omega$ increases. To understand the phenomenon, suppose that the centrifugal force is dropped. The Coriolis force can be replaced by a body force that acts as a traction $t$ at the outlet, pointing downwards at outflow points. This directs the flow towards the bottom of the outlet. This effect is more important the higher $\omega$ is. In order to capture it, we have employed a mesh of $600 Q_{2} / Q_{1}$ elements (with 2501 nodal points) refined near $y=-1$. The velocity field obtained using this mesh and the Galerkin method with $v=1$ is shown in Fig. 4.

The boundary layer created as $\omega$ increases is shown in Fig. 5. The abscissa is measured in grid spacing units, since otherwise the boundary layer is too thin to be observed. It is seen that the Galerkin method presents global oscillations for $v=10^{-4}$ (that are present in the whole computational domain), whereas the ASGS method only presents localized boundary layer oscillations. In fact, for smaller values of $v$ the Galerkin solution is completely oscillatory, whereas the solution obtained with the ASGS method is perfectly smooth for all $v$.

The streamlines and pressure contours computed using the ASGS formulation (incorporating now the centrifugal force) are shown in Fig. 6. Now this result has been obtained using a mesh of $Q_{1}$ elements obtained by splitting each biquadratic element into four bilinear ones and using equal velocity-pressure interpolation. It is observed that the centrifugal force dominates the pressure and also that the condition $p \approx 0$ at the outlet to which (86) reduces when $v$ is very small is well approximated.

\subsection{Flow in a porous cavity}

This last example consists of the solution of the Stokes problem (problem (1)-(3) without the nonlinear convective term) in the T-shaped cavity $\bar{\Omega}=[-4,4] \times[0,6] \cup[-7,7] \times[6,9]$. A parabolic velocity profile $\boldsymbol{u}=\left(u_{x}(y), 0\right)$, with $u_{x}(y)=1-(y / 3-3)^{2}$, is prescribed at the inlet $x=-7$, whereas the outlet $x=7$ is left free (i.e., condition (86) at $x=7$ is applied). The no-slip condition is used for the rest of the boundary, except at $y=9$, where $\boldsymbol{u}=(0,1)$ is prescribed.

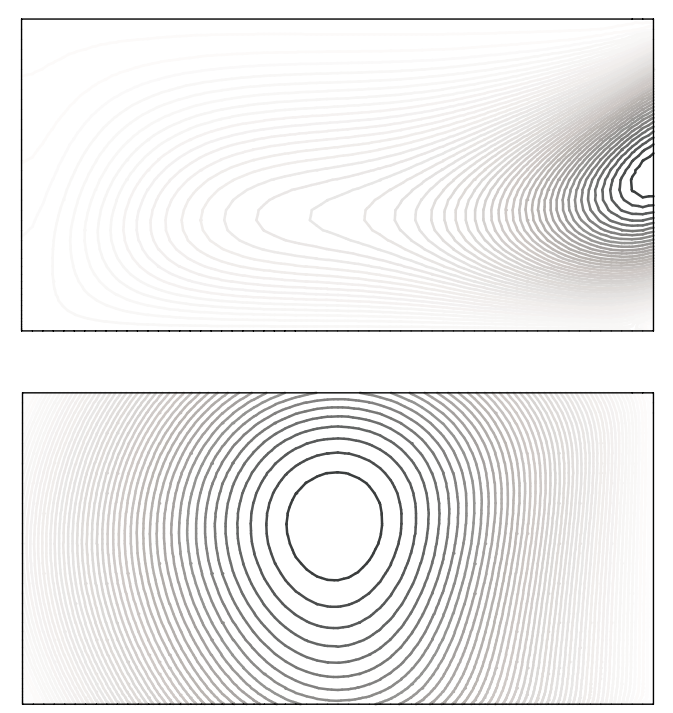

Fig. 6. Streamlines (top) and pressure contours (bottom) for $v=10^{-4}$ using the ASGS method for the rotating Poiseuille flow problem $\left(Q_{1} / Q_{1}\right.$ element $)$. 


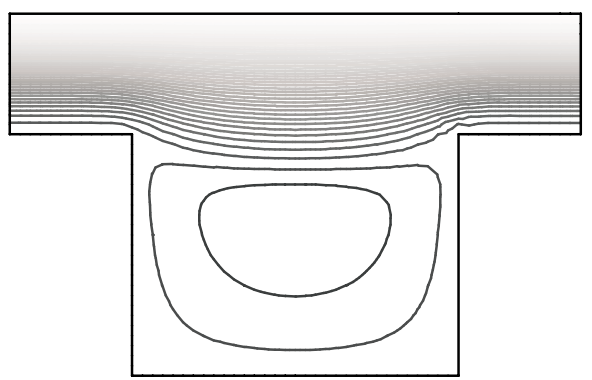

(1)

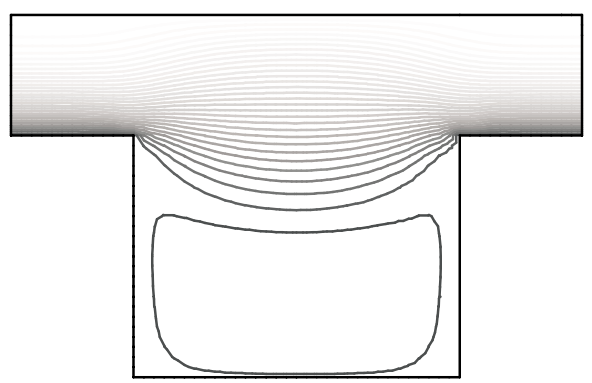

(3)

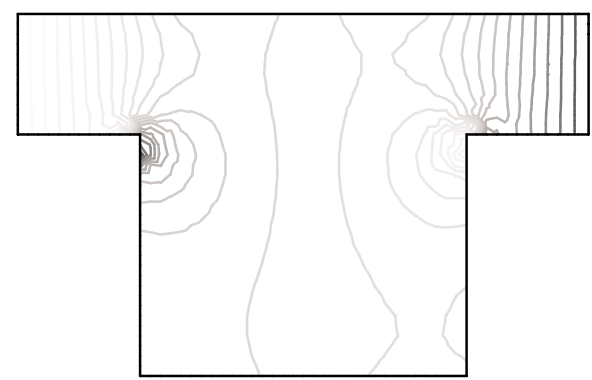

(2)

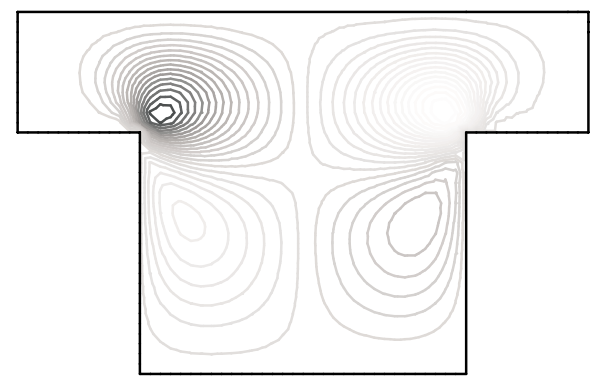

(4)

Fig. 7. Results for the flow in a cavity for $\sigma=0$ and using the Galerkin method ( $P_{2} / P_{1}$ element). (1) Streamlines; (2) pressure contours; (3) $x$-velocity contours; (4) $y$-velocity contours.

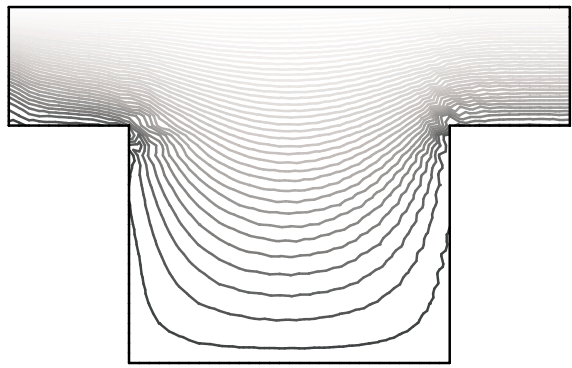

(1)

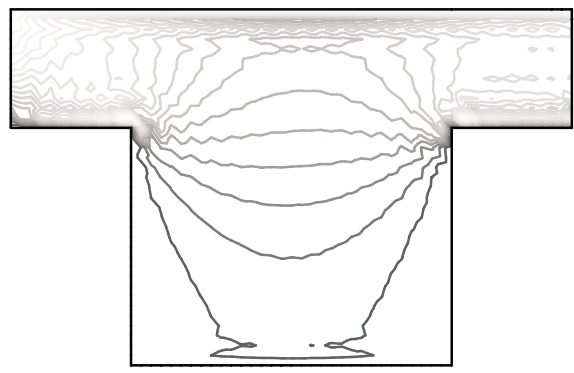

(3)

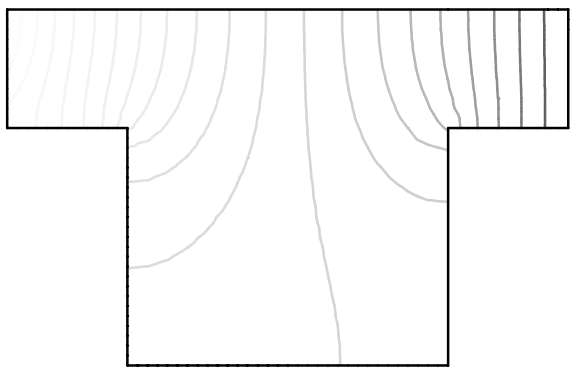

(2)

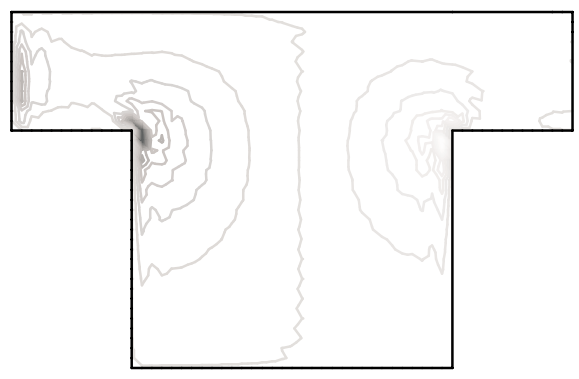

(4)

Fig. 8. Results for the flow in a cavity for $\sigma=10^{4}$ and using the Galerkin method $\left(P_{2} / P_{1}\right.$ element). (1) Streamlines; (2) pressure contours; (3) $x$-velocity contours; (4) $y$-velocity contours.

The domain $\Omega$ is discretized in this case using quadratic triangles $\left(P_{2}\right.$ elements) and equally distributed nodes, separated 0.25 in both the $x$ - and $y$-directions. The total number of nodes is 1533 and the total number of elements 720. Both for the Galerkin and for the ASGS methods, the $P_{2} / P_{1}$ velocity-pressure 


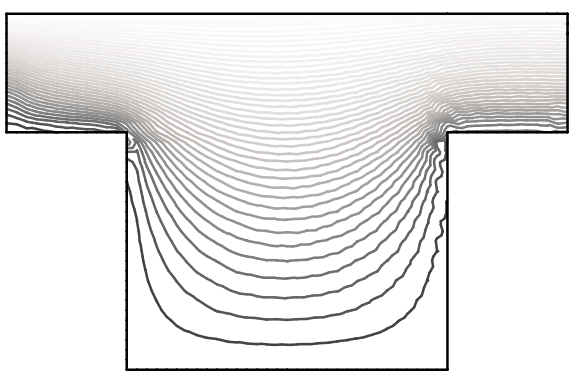

(1)

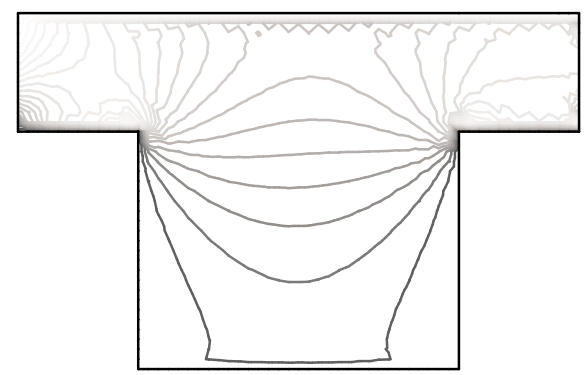

(3)

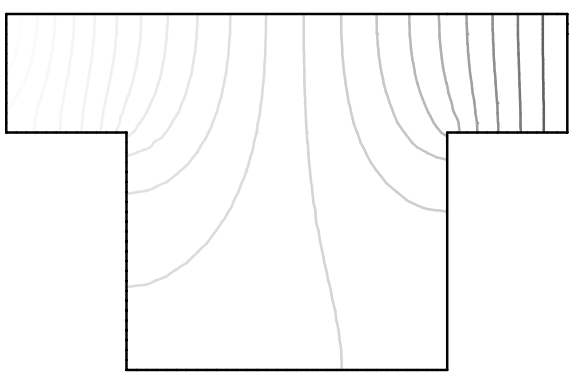

(2)

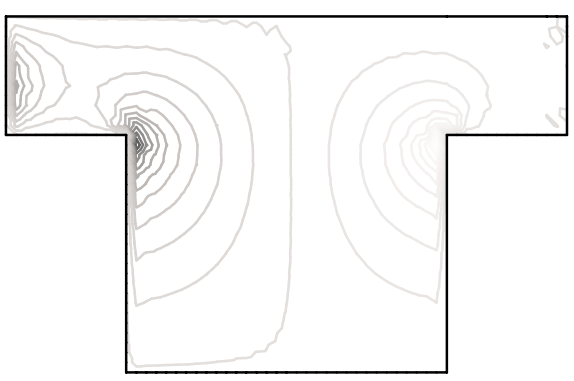

(4)

Fig. 9. Results for the flow in a cavity for $\sigma=10^{4}$ and using the ASGS method ( $P_{2} / P_{1}$ element). (1) Streamlines; (2) pressure contours; (3) $x$-velocity contours; (4) $y$-velocity contours.

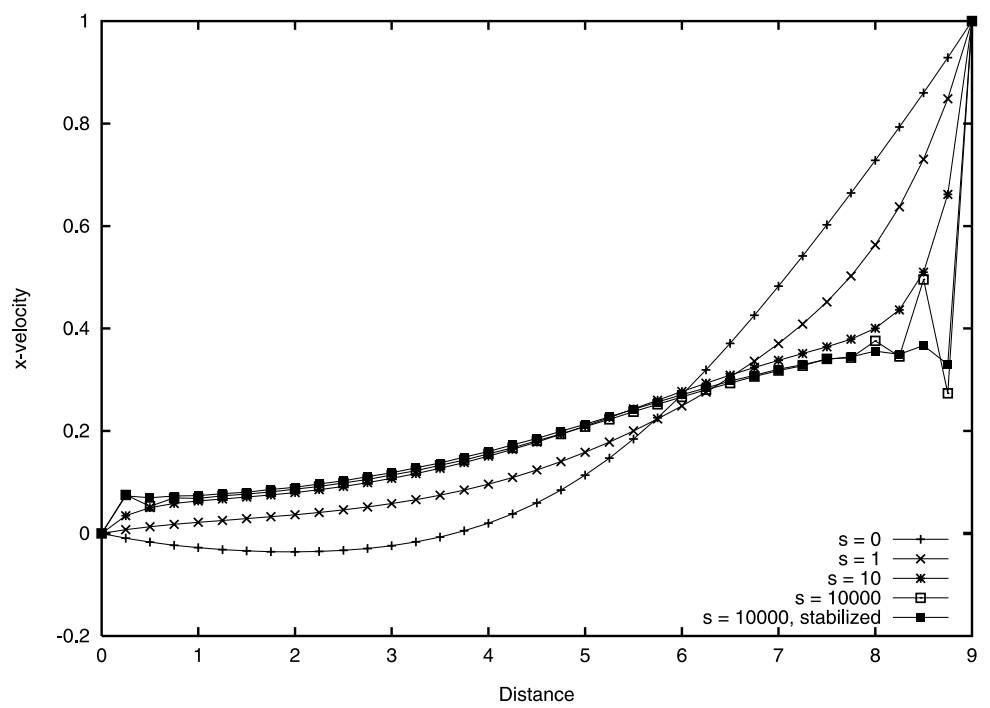

Fig. 10. $x$-velocity profiles at the vertical section $x=0$ of the cavity for different values of the $\sigma$ coefficient (denoted $s$ in the figure). Results using the ASGS method are only shown for $\sigma=10^{4}$.

interpolation has been employed, so that the need for using a stabilization method does not come from the pressure interpolation. The objective of this example is to see how does the ASGS method work when the coefficient $\sigma$ is large. The physical parameters employed in this example are $v=1, \omega=0$ and different values of $\sigma$, up to $10^{4}$.

Numerical results are shown in Figs. 7-9. The former corresponds to the case $\sigma=0$ and the Galerkin results are smooth. However, from Fig. 8 it is seen that when $\sigma=10^{4}$ there are numerical oscillations, higher near the boundaries and propagating towards the interior of the domain. These oscillations are 
partly damped out when the ASGS method is employed (see Fig. 9), even though some of them remain. The improvement can be better observed from Fig. 10, where the $x$-velocity component along the mid-section $x=0$ is plotted. This behavior is exactly what should be expected from the discussion of the previous section.

\section{Conclusions}

In this paper, we have described a finite element method for solving the stationary Navier-Stokes equations written in a rotating frame of reference, incorporating the permeability of the medium and also a small penalty parameter in the continuity equation.

The stabilized formulation employed can be motivated from the sub-grid scale concept, using in particular an algebraic approximation to the sub-scales. The method depends on two algorithmic parameters the form of which has been proposed. For the first of them, $\tau_{1}$, its expression is motivated from the analysis of a simple 1D case. However, this expression is further justified by the convergence analysis presented for the linearized problem, which shows that it leads to optimal error estimates. It is also this analysis who dictates how the second numerical parameter, $\tau_{2}$, must behave. In contrast to other error estimates for stabilized finite element methods, the one presented here relies completely on this parameter $\tau_{2}$.

Limit cases discussed show that the error estimate is optimal and that the stabilized formulation must be able to work equally well for all the possible values of the physical parameters. Numerical experiments presented here show that this is in fact true, provided the algorithmic constants on which the formulation depends are properly chosen.

\section{Acknowledgements}

This work has been partially supported by BRITE-EURAM Project Number BE-1046, entitled Enhanced design of pressure gear pumps using environmentally acceptable hydraulic fluids (ECOPUMP). This support is gratefully acknowledged.

\section{References}

[1] F. Brezzi, M. Fortin, Mixed and Hybrid Finite Element Methods, Springer, Berlin, 1991.

[2] R. Codina, O. Soto, Finite element solution of the Stokes problem with dominating Coriolis force, Computer Methods in Applied Mechanics and Engineering 142 (1997) 215-234.

[3] I. Harari, T.J.R. Hughes, Stabilized finite element methods for steady advection-diffusion with production, Computer Methods in Applied Mechanics and Engineering 115 (1994) 165-191.

[4] S. Idelsohn, N. Nigro, M. Storti, G. Buscaglia, A Petrov-Galerkin formulation for advection-reaction-diffusion problems, Computer Methods in Applied Mechanics and Engineering 136 (1996) 27-46.

[5] T.J.R. Hughes, L.P. Franca, M. Balestra, A new finite element formulation for computational fluid dynamics. V. Circumventing the Babuška-Brezzi condition a stable Petrov-Galerkin formulation for the Stokes problem accommodating equal-order interpolations, Computer Methods in Applied Mechanics and Engineering 59 (1986) 85-99.

[6] L.P. Franca, S.L. Frey, Stabilized finite element methods. II. The incompressible Navier-Stokes equations, Computer Methods in Applied Mechanics and Engineering 99 (1992) 209-233.

[7] M. Storti, N. Nigro, S. Idelsohn, Equal-order interpolations a unified approach to stabilize the incompressible and advective effects, Computer Methods in Applied Mechanics and Engineering 143 (1997) 317-331.

[8] L.P. Franca, T.J.R. Hughes, Convergence analyses of Galerkin least-squares methods for advective-diffusive forms of the Stokes and incompressible Navier-Stokes equations, Computer Methods in Applied Mechanics and Engineering 105 (1993) $285-298$.

[9] P. Hansbo, A. Szepessy, A velocity-pressure streamline diffusion finite element method for the incompressible Navier-Stokes equations, Computer Methods in Applied Mechanics and Engineering 84 (1990) 175-192.

[10] L. Tobiska, G. Lube, A modified streamline-diffusion method for solving the stationary Navier-Stokes equations, Numerische Mathematik 59 (1991) 13-29.

[11] L. Tobiska, R. Verfürth, Analysis of a streamline diffusion finite element method for the Stokes and Navier-Stokes equations, SIAM Journal on Numerical Analysis 33 (1996) 107-127.

[12] T.J.R. Hughes, Multiscale phenomena, Green's function, the Dirichlet-to-Neumann formulation, subgrid scale models bubbles and the origins of stabilized formulations, Computer Methods in Applied Mechanics and Engineering 127 (1995) 387-401. 
[13] R. Codina, Comparison of some finite element methods for solving the diffusion-convection-reaction equation, Computer Methods in Applied Mechanics and Engineering 156 (1998) 185-210.

[14] T.J.R. Hughes, M. Mallet, A new finite element method for computational fluid dynamics. III The generalized streamline operator for multidimensional advective-diffusive systems, Computer Methods in Applied Mechanics and Engineering 58 (1986) 305-328.

[15] T.J.R. Hughes, L.P. Franca, G.M. Hulbert, A new finite element formulation for computational fluid dynamics. VIII. The Galerkin/least-squares method for advective-diffusive equations, Computer Methods in Applied Mechanics and Engineering 73 (1989) 173-189.

[16] R. Codina, On stabilized finite element methods for linear systems of convection-diffusion-reaction equations, Computer Methods in Applied Mechanics and Engineering 188 (2000) 61-82.

[17] L. Franca, S.L. Frey, T.J.R. Hughes, Stabilized finite element methods. I. Application to the advective-diffusive model, Computer Methods in Applied Mechanics and Engineering 95 (1992) 253-276.

[18] L.P. Franca, C. Farhat, Bubble functions prompt unusual stabilized finite element methods, Computer Methods in Applied Mechanics and Engineering 123 (1994) 299-308.

[19] S.C. Brenner, L.R. Scott, The Mathematical Theory of Finite Element Methods, Springer, Berlin, 1994.

[20] L. Franca, R. Stenberg, Error analysis of some Galerkin least-squares methods for the elasticity equations, SIAM Journal on Numerical Analysis 28 (1991) 1680-1697.

[21] F. Brezzi, J. Douglas, Stabilized mixed methods for the Stokes problem, Numerische Mathematik 53 (1988) $225-235$.

[22] R. Codina, Numerical solution of the incompressible Navier-Stokes equations with Coriolis forces based on the discretization of the total time derivative, Journal of Computational Physics 148 (1999) 467-496. 